\title{
Palladium-Catalyzed Direct Functionalization of Imidazolinone: Synthesis of Dibromophakellstatin
}

\author{
Jianming Lu, Xianghui Tan and Chuo Chen*
}

Supporting Information

General Comments $\quad$ S1

$\begin{array}{ll}\text { Mechanistic Studies } & \text { S2 }\end{array}$

$\begin{array}{ll}\text { Computational Details } & \text { S4 }\end{array}$

Experimental Section $\quad$ S9

$\begin{array}{ll}\text { NMR Spectra } & \text { S17 }\end{array}$

\section{General Comments}

We have examined the direct arylation of imidazolinone (1) with 6 different palladium sources, 15 different phosphine ligands, 12 different bases and 14 different solvent systems. There is no significant difference between using $\mathrm{Pd}(\mathrm{OAc})_{2}$ and $\mathrm{Pd}(\mathrm{TFA})_{2}$. This reaction proceeds less efficiently or reproducibly with $\mathrm{Pd}_{2}(\mathrm{dba})_{3} /(o$-biphenyl $) \mathrm{PCy}_{2}, \mathrm{O}=\mathrm{PCy}_{3}, \mathrm{O}=\mathrm{PPh}_{3}, \mathrm{P}(\mathrm{OMe})_{3}, \mathrm{PCy}_{3} \cdot \mathrm{HBF}_{4}, \mathrm{PCy}_{3}$, $\mathrm{P}^{\mathrm{n}} \mathrm{Bu}_{3} / \mathrm{DMF}, \mathrm{NMP} / \mathrm{Na}_{2} \mathrm{CO}_{3}$.

Aryl bromides are less reactive; however, good results can be obtained with electronic-deficient aryl bromides. The electron-rich aryl bromides only react slowly. We suspect that the rate-limiting step shifts to oxidative addition of palladium $(0)$ to aryl bromide in these cases.

For the synthesis of dibromophakellstatin, phthalimide 11 can be prepared in DMSO and used in situ for the subsequent vinylation reaction. However, removal of the potassium bromide salt improves the efficiency of the palladium-catalyzed vinylation reaction. We have found that $\mathbf{1 2}$ decomposed upon prolonged heating, and 13 was obtained in only $25 \%$ overall yield using the standard protocol. Therefore, 1 was used in large excess to decrease the reaction time. Unreacted imidazolinone 1 can be recovered from washing the reaction mixture with water directly or through a short pad of reversephase silica gel. Partial removal of palladium black with reverse-phase silica gel reduces the adsorption problem and increases the overall yield. After hydrogenation, the remaining palladium black can be easily filtered off. The diminished overall yield of $\mathbf{1 3}$ is due to the adsorption of reaction products by palladium. The one-pot hydrogenation/phthalimide deprotection method gave $\sim 30 \%$ yield of 13. 


\section{Mechanistic Studies}

Scheme S1 summarizes the experimentally excluded mechanisms. Additional evidences against the anti- $\beta$-H elimination, $\alpha$-elimination and electrophilic palladation pathway are also presented below.

\section{Scheme S1.}

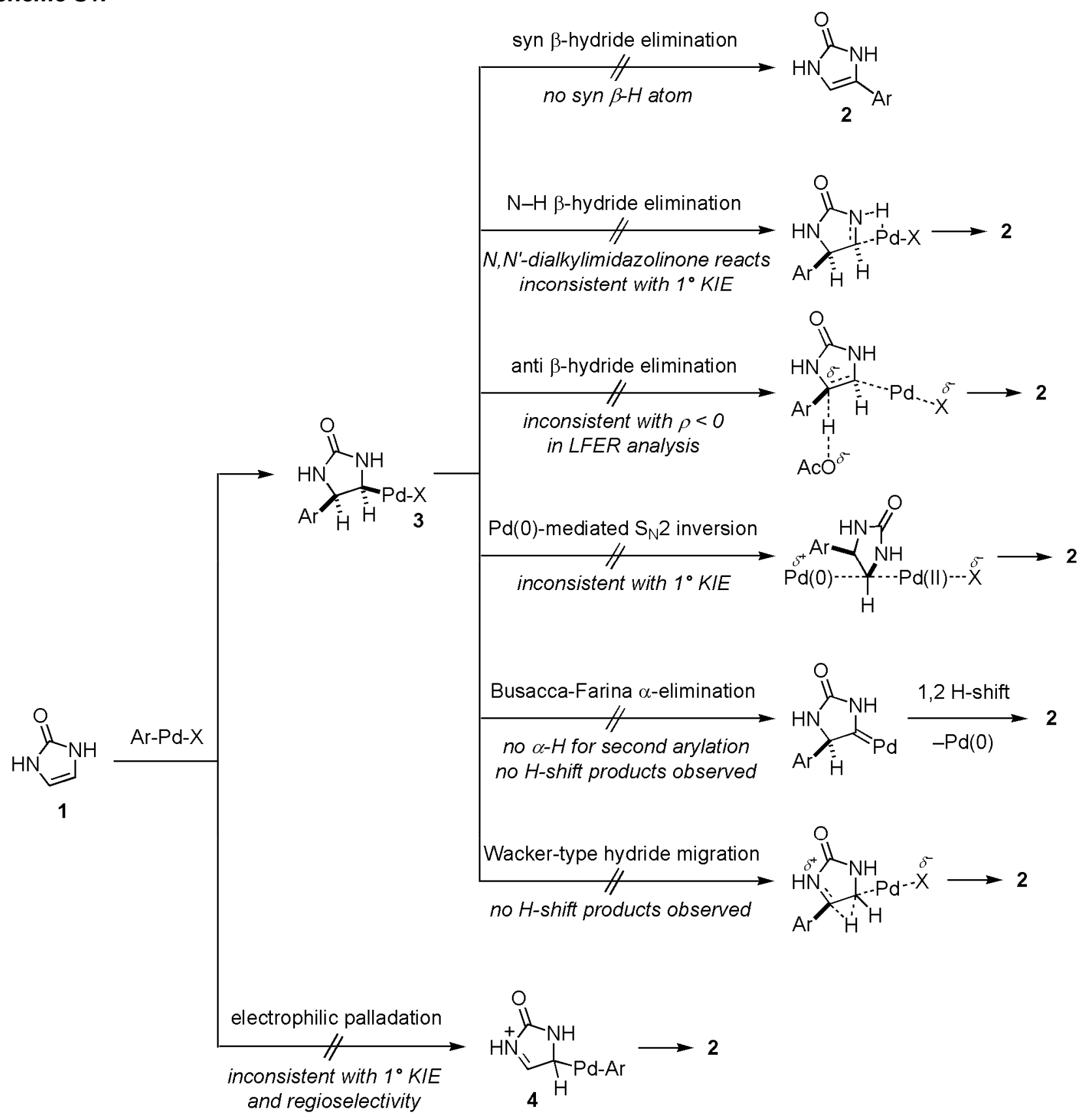

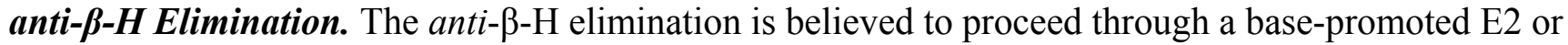
$\mathrm{E} 1_{\mathrm{cb}}$ mechanism (Scheme $\mathrm{S} 2$ ). An electron-rich or neutral center adjacent to the aryl group in 14 is therefore expected to form, with $\rho \geq 0$ in the linear free-energy relationship (LFER) analysis. However, the LFER analysis suggests that an electron-deficient center $(\rho=-1.072)$ adjacent to the aryl group was developed in the transition state (Table S1, Figure S1). The reaction proceeded cleanly without detectable side-products and intermediates for the aryl iodides used in Table S2. 
Scheme S2.

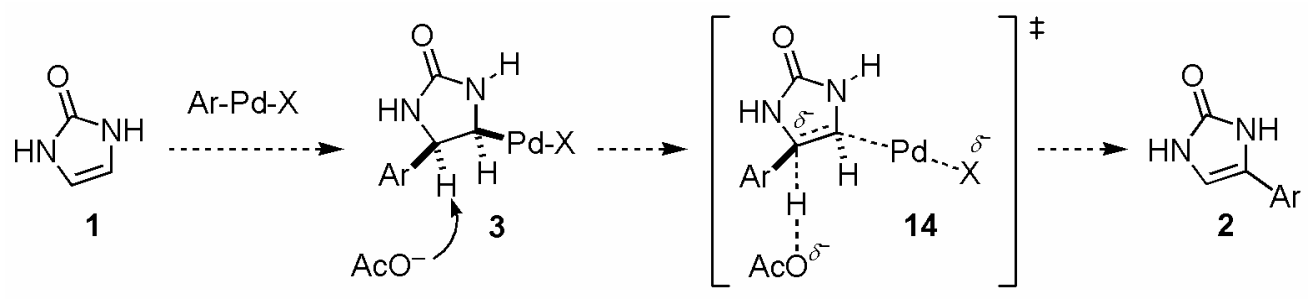

Table S1. The linear free-energy relationship analysis of arylation of 1.

\begin{tabular}{|c|c|c|c|c|c|c|c|c|}
\hline \multirow{2}{*}{$A r-1$} & \multicolumn{4}{|c|}{$\%$ product $^{*}$} & \multirow{2}{*}{ slope } & \multirow{2}{*}{$r$} & \multirow{2}{*}{$k_{\text {rel }}$} & \multirow[b]{2}{*}{$\sigma$} \\
\hline & $3 \mathrm{~min}$ & $5 \mathrm{~min}$ & $10 \mathrm{~min}$ & $20 \mathrm{~min}$ & & & & \\
\hline $3-\mathrm{NO}_{2}-\mathrm{C}_{6} \mathrm{H}_{4}-\mathrm{I}$ & $2.1^{\dagger}$ & 2.1 & 3.7 & 4.2 & 0.134 & 0.921 & 0.161 & $0.71 \ddagger$ \\
\hline $4-\mathrm{CN}-\mathrm{C}_{6} \mathrm{H}_{4}-\mathrm{I}$ & - & $\sim 0$ & 1.6 & 3.2 & 0.206 & 0.982 & 0.246 & $0.66 \ddagger$ \\
\hline $\mathrm{H}-\mathrm{C}_{6} \mathrm{H}_{4}-\mathrm{I}$ & 5.2 & 8.2 & 12.7 & 19.2 & 0.836 & 0.993 & 1.000 & 0 \\
\hline $4-\mathrm{Me}-\mathrm{C}_{6} \mathrm{H}_{4}-\mathrm{I}$ & 6.3 & 9.4 & 16.4 & 22.3 & 0.920 & 0.973 & 1.100 & $-0.17 \ddagger$ \\
\hline $4-\mathrm{OMe}-\mathrm{C}_{6} \mathrm{H}_{4}-\mathrm{I}$ & 7.7 & 11.3 & 14.7 & 25.6 & 1.013 & 0.995 & 1.212 & $-0.27 \ddagger$ \\
\hline
\end{tabular}

* Determined by NMR with $10 \mathrm{~s}$ recycle delay. ${ }^{\dagger}$ Sample taken at $3.5 \mathrm{~min} .{ }^{*}$ Data taken from Lowry, T. H.; Richardson, K. S. Mechanism and Theory in Organic Chemistry, 3rd ed. HarperCollins: New York, 1987; pp 144.
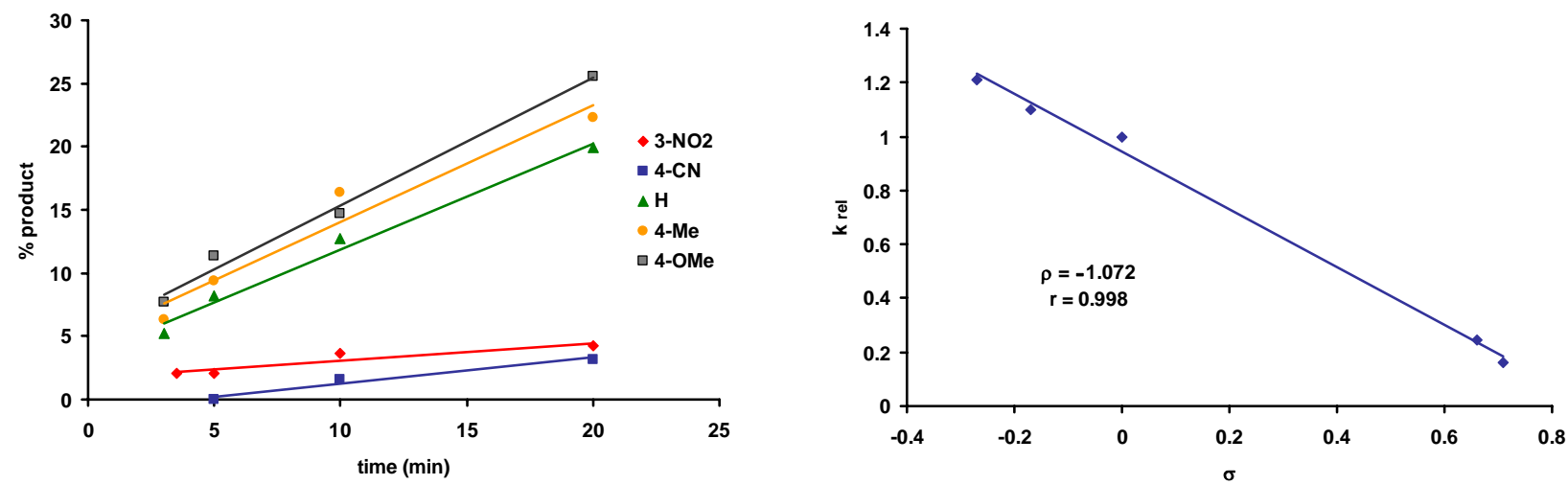

Figure S1. The Hammett linear free-energy relationship analysis.

$\boldsymbol{\alpha}$-Elimination. In addition to the absence of $\mathrm{H} / \mathrm{D}$ migration products with $8-d_{1}$ (Scheme 4 ), the $\alpha$ elimination mechanism can not explain the formation of the bisarylation products. During the second arylation step, there is no $\alpha-\mathrm{H}$ available for the elimination in $\mathbf{1 5}$ (Scheme S3).

Scheme S3.

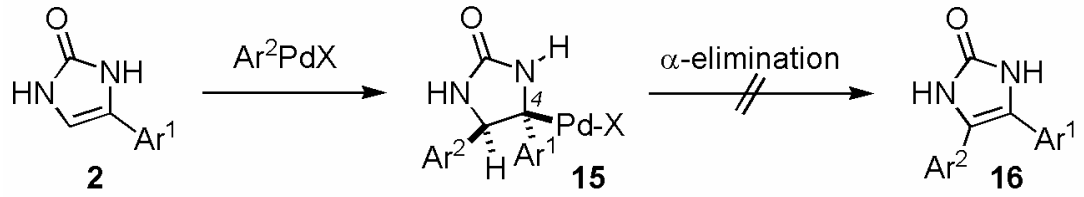

Electrophilic Palladation. In addition to the arylation of 8, arylation of $\mathbf{1 7}$ also occurs preferentially at the less electron-rich 5-position (Scheme S4). The regioselectivity is opposite to the electrophilic formylation of 17. 


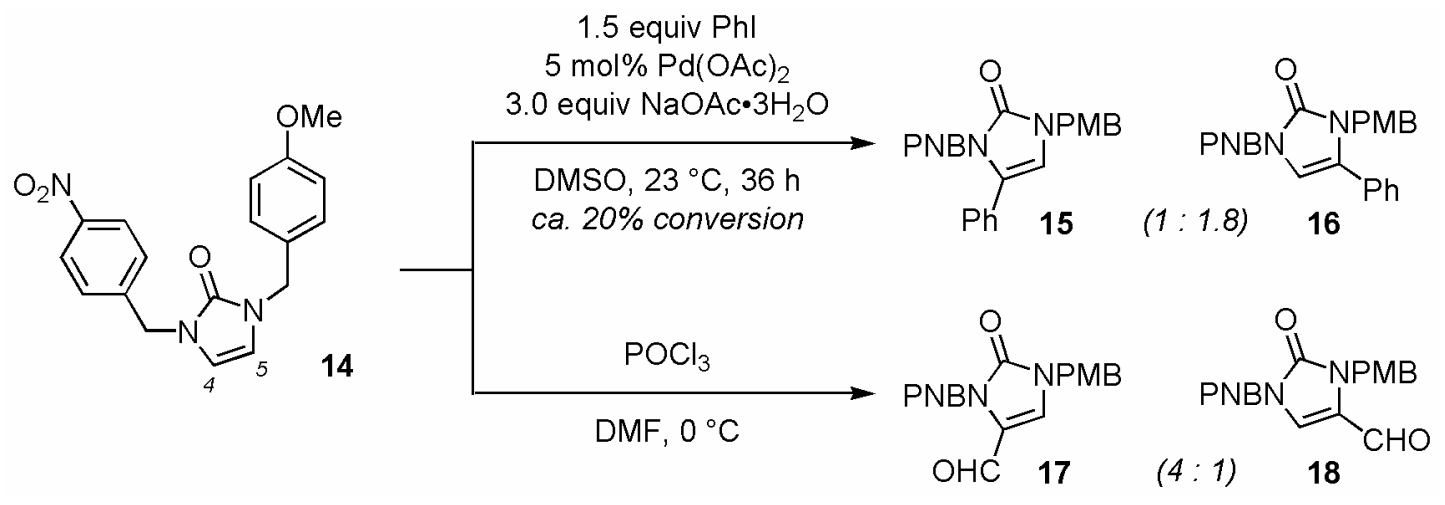

\section{Computational Studies}

The activation barriers for the acetate ligand-assisted $\mathrm{C}-\mathrm{H}$ insertion of the cis- and trans- $\mathrm{PdL}_{2} \mathrm{X}_{2}$ complex (Scheme S5, Table S2) were evaluated. All the computations were performed on Jaguar 6.5 suite $^{\mathrm{S} 1}$ using the B3LYP functional and LACVP**++ basis set. ${ }^{\mathrm{S}, 3}$ All stationary points were subjected to frequency analysis and characterized as a minimum (no imaginary frequencies) or as a saddle point (single imaginary frequency) on the potential energy surface. Transition states were verified by imaginary frequency animation with desired vibrational modes. The single-point energy and solvation energy (DMSO, dielectric constant: 47.240 , probe radius: $2.41 \AA$ ) calculations were then performed on the gas-phase optimized geometries. The solvation was treated with a self-consistent reaction field (SCRF) method, using its own Poisson-Boltzmann solver. ${ }^{\mathrm{S}, 5}$ Zero-point-energy (ZPE) and entropy corrections were derived using unscaled frequencies. Thermodynamic properties were calculated assuming ideal gas at $298.15 \mathrm{~K}$. The resulting activation barrier for the cis- $\mathrm{PdL}_{2} \mathrm{X}_{2}$ complex $\left(\Delta \mathrm{H}_{\mathrm{DMSO}, 298.15 \mathrm{~K}}^{\ddagger}=17.9 \mathrm{kcal} / \mathrm{mol}\right)$ is comparable to the reported activation barriers for the Wacker oxidation $\left(\Delta \mathrm{H}^{\ddagger}{ }_{\mathrm{H} 2 \mathrm{O}, 0 \mathrm{~K}}=12.5-18.8 \mathrm{kcal} / \mathrm{mol}\right)$ calculated by the same method. ${ }^{\mathrm{S} 3}$

Scheme S5.

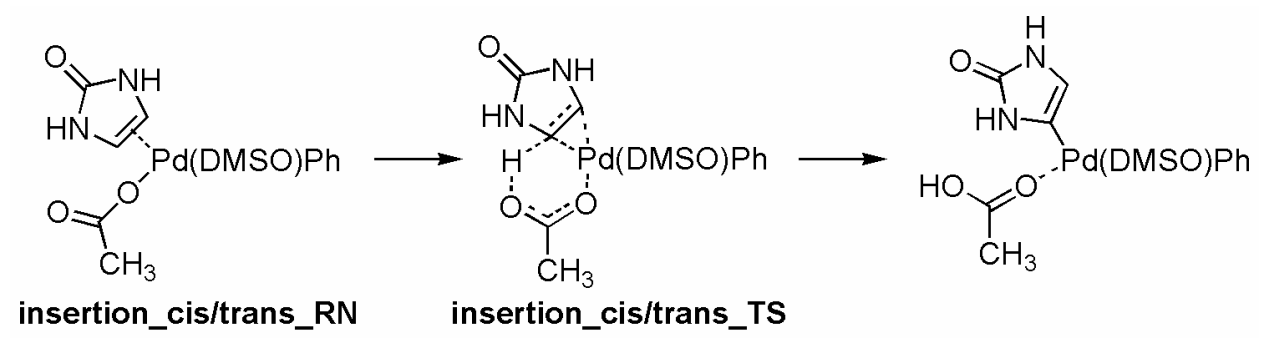

Table S2. The computed energies for the $\mathrm{C}-\mathrm{H}$ insertion pathway.

\begin{tabular}{cccccccc}
\hline & $\begin{array}{c}\text { SCFE(gas) } \\
\text { (hartree) }\end{array}$ & $\begin{array}{c}\text { ZPE* }^{*} \\
(\mathrm{kcal} / \mathrm{mol})\end{array}$ & $\begin{array}{c}\text { solvation* } \\
(\mathrm{kcal} / \mathrm{mol})\end{array}$ & $\begin{array}{c}\mathrm{H}(\mathrm{gas}) \\
\text { (hartree) }\end{array}$ & $\begin{array}{c}\Delta \mathrm{H}(\mathrm{soln})^{*} \\
(\mathrm{kcal} / \mathrm{mol})\end{array}$ & $\begin{array}{c}\mathrm{G}(\mathrm{gas}) \\
\text { (hartree) }\end{array}$ & $\begin{array}{c}\Delta \mathrm{G}(\mathrm{soln})^{*} \\
(\mathrm{kcal} / \mathrm{mol})\end{array}$ \\
\hline insertion_cis_RN & -1441.628703 & 188.34 & -29.217 & -1441.303087 & 0 & -1441.385285 & 0 \\
insertion_cis_TS & -1441.599126 & 185.41 & -26.648 & -1441.278736 & 17.85 & -1441.360336 & 18.23 \\
\hline insertion_trans_RN & -1441.630662 & 188.36 & -31.219 & -1441.305082 & 0 & -1441.387571 & 0 \\
insertion_trans_TS & -1441.586045 & 184.63 & -27.597 & -1441.266744 & 27.68 & -1441.348879 & 27.90 \\
\hline
\end{tabular}

* Converting unit: 1 hartree $=627.50947093 \mathrm{kcal} / \mathrm{mol}$. 

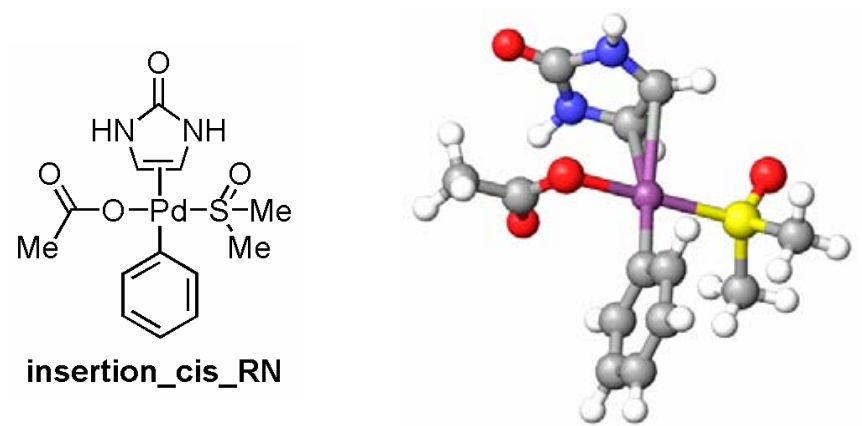

Gas-phase SCF energy: -1441.62870306573 hartrees

Solvation energy: -0.04656015308 hartrees

Zero point energy (ZPE): $188.343560 \mathrm{kcal} / \mathrm{mol}$

$\mathrm{T}=298.15 \mathrm{~K}$

Total internal energy, Utot (SCFE + ZPE + U) : -1441.304032 hartrees

Total enthalpy, Htot (Utot + pV) : -1441.303087 hartrees

Total Gibbs free energy, Gtot (Htot - T*S) : -1441.385285 hartrees

Geometry Coordinates

\begin{tabular}{|c|c|}
\hline atom & $\mathrm{x}$ \\
\hline $\mathrm{Pd} 1$ & 0.1134567092 \\
\hline $\mathrm{C} 2$ & 0.0869390083 \\
\hline $\mathrm{C} 3$ & 1.4375253368 \\
\hline $\mathrm{H} 4$ & 2.1221518323 \\
\hline N5 & 1.8526043140 \\
\hline H6 & 2.7322522031 \\
\hline N7 & -0.3056823555 \\
\hline H8 & -1.2518250773 \\
\hline $\mathrm{C9}$ & 0.7617067172 \\
\hline 010 & 0.7524659286 \\
\hline 011 & 0.0785928763 \\
\hline C12 & -1.0617479672 \\
\hline 013 & -2.0499239689 \\
\hline $\mathrm{H} 14$ & -0.5320891220 \\
\hline C15 & -1.0658448109 \\
\hline $\mathrm{H} 16$ & -0.5734663728 \\
\hline $\mathrm{H} 17$ & -2.0904807347 \\
\hline $\mathrm{H} 18$ & -0.5016421687 \\
\hline C19 & -0.4665371925 \\
\hline $\mathrm{C} 20$ & -1.3146829351 \\
\hline $\mathrm{C} 21$ & 0.4755510173 \\
\hline $\mathrm{C} 22$ & -1.8333355418 \\
\hline $\mathrm{C} 23$ & -2.2532637938 \\
\hline $\mathrm{C} 24$ & 0.0496468295 \\
\hline H2 5 & 1.5401757784 \\
\hline H2 6 & -2.5679038715 \\
\hline H27 & -3.3159103122 \\
\hline $\mathrm{H} 28$ & 0.7873012772 \\
\hline H29 & -1.6434807042 \\
\hline S30 & 0.2272873294 \\
\hline 031 & 0.7090725170 \\
\hline $\mathrm{C} 32$ & -1.3861918170 \\
\hline H33 & -1.7152873223 \\
\hline H34 & -1.2744277047 \\
\hline H35 & -2.0797938862 \\
\hline C36 & 1.2278496965 \\
\hline H37 & 0.8438220418 \\
\hline
\end{tabular}

angstroms

\begin{tabular}{rr} 
y & \multicolumn{1}{c}{$z$} \\
0.0194329846 & -0.0428223416 \\
-0.0130484491 & 2.4923242386 \\
-0.0748698712 & 2.2442450199 \\
0.7377528254 & 2.0548409058 \\
-1.3585117929 & 2.5634951536 \\
-1.7802082453 & 2.3057177336 \\
-1.2568159414 & 2.9779880706 \\
-1.6074726142 & 2.8826173957 \\
-2.1531977032 & 2.9361286730 \\
-3.3509728495 & 3.1719387466 \\
-2.0400916121 & -0.1046110160 \\
-2.5858611883 & 0.2231914783 \\
-1.9554407197 & 0.6325443754 \\
0.8651009891 & 2.5894273917 \\
-4.1019847214 & 0.1167864231 \\
-4.4261512123 & -0.8037683746 \\
-4.4754973399 & 0.1560208579 \\
-4.5110189451 & 0.9624491665 \\
-0.0361942897 & -1.9793992131 \\
-0.1923204264 & -4.6507152745 \\
-0.0833436430 & -3.0133168167 \\
-0.0935663983 & -2.2788022675 \\
-0.1659482770 & -3.6146043157 \\
-0.1583323199 & -4.3474182631 \\
-0.0867190315 & -2.7933604803 \\
-0.1226028277 & -1.4790413859 \\
-0.2175029150 & -3.8392774911 \\
-0.2017041135 & -5.1451957233 \\
-0.2557700475 & -5.6843321342 \\
2.3491083769 & -0.0745432498 \\
2.9796697533 & 1.2096394578 \\
3.0773508262 & -0.4892557438 \\
2.6913482139 & -1.4559292022 \\
4.1642130896 & -0.4949993946 \\
2.7714720131 & 0.2956948364 \\
3.0236390797 & -1.4352687420 \\
2.6502021987 & -2.3860173880
\end{tabular}



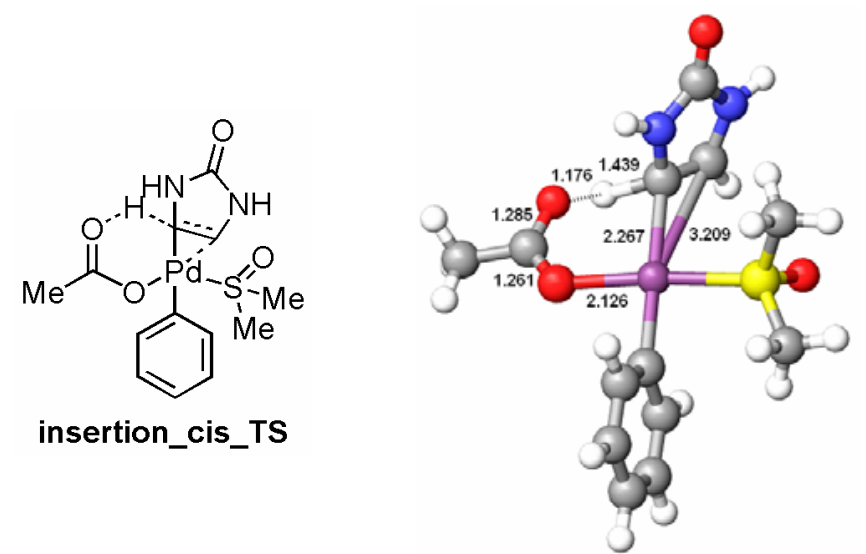

Imaginary frequency: $-815.66 \mathrm{~cm}^{\wedge}-1$

Gas-phase SCF energy: -1441. 59912583532 hartrees

Solvation energy: -0.04246695678 hartrees

zero point energy (ZPE) : $185.407163 \mathrm{kcal} / \mathrm{mol}$

$\mathrm{T}=298.15 \mathrm{~K}$

Total internal energy, Utot (SCFE + ZPE + U): -1441.279680 hartrees Total enthalpy, Htot (Utot + pV): -1441.278736 hartrees

Total Gibbs free energy, Gtot (Htot - $T^{*}$ ) : -1441.360336 hartrees

Geometry Coordinates

$\begin{array}{lc}\text { atom } & \text { X } \\ \text { Pd1 } & -1.6663957445 \\ \text { C2 } & -2.4498980561 \\ \text { C3 } & -3.6887129598 \\ \text { H4 } & -4.1853663771 \\ \text { N5 } & -4.2980541029 \\ \text { H6 } & -5.1894529967 \\ \text { N7 } & -2.3605736915 \\ \text { H8 } & -1.5396230956 \\ \text { C9 } & -3.4619253523 \\ \text { O10 } & -3.6850322051 \\ \text { O11 } & 0.2616881107 \\ \text { C12 } & 0.4871208563 \\ \text { O13 } & -0.3738589216 \\ \text { H14 } & -1.3389791992 \\ \text { C15 } & 1.8539781350 \\ \text { H16 } & 2.4978522691 \\ \text { H17 } & 1.7511591594 \\ \text { H18 } & 2.2952192706 \\ \text { C19 } & -0.9585450688 \\ \text { C20 } & 0.1294429518 \\ \text { C21 } & -0.0669430728 \\ \text { C22 } & -1.2955415905 \\ \text { C23 } & -1.7517620940 \\ \text { C24 } & 0.4725252967 \\ \text { H25 } & 0.2285804640 \\ \text { H26 } & -1.9768757124 \\ \text { H27 } & -16167638789 \\ \text { H28 } & \\ \text { H29 } & \\ & -5510291867\end{array}$

angstroms

$$
\text { Y }
$$

$-0.5430179393$

0.6005035760

0.3387242415

$-0.6125210945$

1.5215378620

1.6313476658

2.0238123185

2.5681935746

2. 6139868986

3.8000605471

$-0.8866547602$

$-0.8427621410$

$-0.4788455515$

$-0.0746185342$

$-1.2343253580$

$-1.5302769561$

$-2.0598827581$

$-0.3914628973$

$-1.6170230064$

$-3.1685740701$

$-1.0462561043$

$-2.9711748693$

$-3.7447924727$

$-1.8188307395$

$-0.0037438100$

$-3.4380740591$

$-4.7962844667$

$-1.3652300753$

$-3.7674388353$
$\mathrm{Z}$
$-0.5688994996$
1.2243173769
1. 7616212674
1.8826204666
2.1369438913
2. 5966806774
1.2905909558
1.0750577004
1.8781573683
2.1101303677
0.2574943248
1.4975322279
2. 3785805104
1.8420437521
2. 0036527102
1.1755854200
2.7146308679
2. 5445841401
$-2.1478155935$
$-4.2276011185$
$-3.0637281073$
$-2.2724373097$
$-3.3078698595$
$-4.1017057350$
$-2.9694357605$
$-1.5640333777$
$-3.3906248652$
$-4.8057880038$
$-5.0302352313$ 


$\begin{array}{llll}\text { S30 } & -3.7250811180 & -0.2581185142 & -1.6359328341 \\ \text { O31 } & -4.9149523996 & -0.9510557576 & -1.0303707674 \\ \text { C32 } & -3.7233123652 & -0.6589862522 & -3.4102574553 \\ \text { H33 } & -4.7095774065 & -0.3940349036 & -3.7982015140 \\ \text { H34 } & -3.5464024463 & -1.7304304892 & -3.4975660182 \\ \text { H35 } & -2.9229551695 & -0.1112595633 & -3.9107527617 \\ \text { C36 } & -4.0901224859 & 1.5201670494 & -1.7511664213 \\ \text { H37 } & -3.2822414351 & 2.0172459116 & -2.2932618425 \\ \text { H38 } & -4.1562381272 & 1.9079668825 & -0.7357831104 \\ \text { H39 } & -5.0463417144 & 1.6293351908 & -2.2685646740\end{array}$

\section{Summary of the activation energies for cis- $\mathrm{PdL}_{2} \mathrm{X}_{2}$ :}

$\triangle \mathrm{SCFE}$ (gas) : $18.56 \mathrm{kCal} / \mathrm{mol}$ $\triangle \mathrm{ZPE}:-2.94 \mathrm{kcal} / \mathrm{mol}$

$\Delta \mathrm{H}($ gas $): 15.28 \mathrm{kcal} / \mathrm{mol}$

$\Delta \mathrm{G}($ gas $): 15.66 \mathrm{kcal} / \mathrm{mol}$

Solvation correction : $2.57 \mathrm{kcal} / \mathrm{mol}$

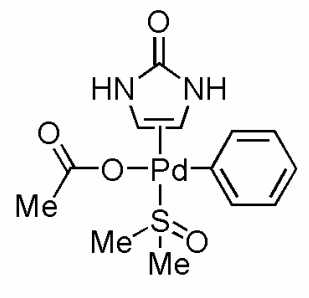

insertion_trans_RN

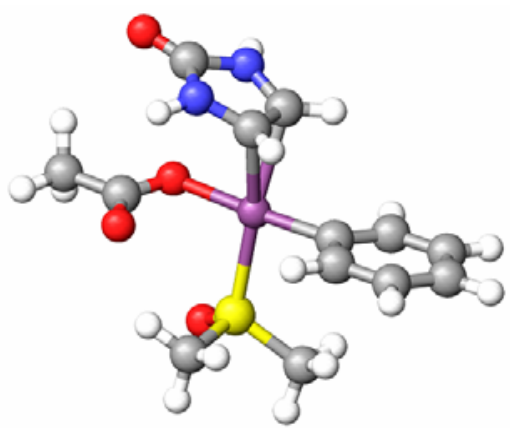

Gas-phase SCF energy: -1441.6306621509 hartrees

Solvation energy: -0.04975049544 hartrees

Zero point energy (ZPE): $188.363 \mathrm{kcal} / \mathrm{mol}$

$\mathrm{T}=298.15 \mathrm{~K}$

Total internal energy, Utot (SCFE + ZPE + U) : -1441.306026 hartrees Total enthalpy, Htot (Utot + pV) : -1441.305082 hartrees

Total Gibbs free energy, Gtot (Htot - $T^{\star} S$ ) : -1441.387571 hartrees

Geometry Coordinates

\begin{tabular}{lrrr} 
atom & $\mathrm{x}$ & \multicolumn{1}{c}{$\mathrm{y}$} & \multicolumn{1}{c}{$\mathrm{z}$} \\
Pd1 & 1.6907251180 & 0.4131613136 & -0.1456261863 \\
C2 & 3.0553979632 & -0.1920012618 & 1.6242202339 \\
C3 & 3.4944748028 & 1.0724712461 & 1.2269473000 \\
H4 & 4.2361693122 & 1.3109136451 & 0.4792801296 \\
N5 & 3.1587325603 & 1.9526347508 & 2.2405298794 \\
H6 & 3.1024404036 & 2.9565318134 & 2.1452396174 \\
N7 & 2.4661087546 & -0.0438891859 & 2.8701898720 \\
H8 & 1.8046558795 & -0.6999367476 & 3.2648116456 \\
C9 & 2.4135696306 & 1.3057452443 & 3.2367566618 \\
O10 & 1.8730963918 & 1.7990524710 & 4.2068389561 \\
O11 & 0.3862858819 & 1.4516825549 & 1.1715632501 \\
C12 & -0.5110288138 & 0.7139237999 & 1.7453906475 \\
O13 & -0.5905298225 & -0.5232406243 & 1.5937857807 \\
H14 & 3.3996443564 & -1.1515018060 & 1.2684641654 \\
C15 & -1.4682965064 & 1.4639446809 & 2.6561747646 \\
H16 & -0.9023014391 & 1.9356861554 & 3.4663765477 \\
H17 & -2.2112083139 & 0.7817069919 & 3.0728399442 \\
H18 & -1.9642511163 & 2.2604775644 & 2.0928549160
\end{tabular}




$\begin{array}{lrrr}\text { C19 } & 3.0133288585 & -0.4731290782 & -1.4041440927 \\ \text { C20 } & 4.8632485959 & -1.6905594812 & -3.1642782749 \\ \text { C21 } & 3.1449951607 & -1.8710408983 & -1.4586758915 \\ \text { C22 } & 3.8276137685 & 0.3038573453 & -2.2451308902 \\ \text { C23 } & 4.7447770608 & -0.2981674513 & -3.1173837368 \\ \text { C24 } & 4.0605215803 & -2.4759565149 & -2.3319268001 \\ \text { H25 } & 2.5312200311 & -2.5047372870 & -0.8206295492 \\ \text { H26 } & 3.7479185370 & 1.3893684830 & -2.2346708050 \\ \text { H27 } & 5.3647460412 & 0.3229285990 & -3.7595737039 \\ \text { H28 } & 4.1456229868 & -3.5597050283 & -2.3573642489 \\ \text { H29 } & 5.5746373159 & -2.1574782369 & -3.8395941006 \\ \text { S30 } & -0.0881033316 & 0.2587798449 & -1.8054875244 \\ \text { O31 } & -1.1128880883 & 1.3560480835 & -1.8564962394 \\ \text { C32 } & 0.4805077278 & -0.0254495899 & -3.5130503880 \\ \text { H33 } & 1.1648614188 & -0.8742704806 & -3.5445655484 \\ \text { H34 } & 0.9929152206 & 0.8837190333 & -3.8306017509 \\ \text { H35 } & -0.4094039084 & -0.1913731550 & -4.1247218661 \\ \text { C36 } & -0.9316505758 & -1.3197088832 & -1.4826312084 \\ \text { H37 } & -0.2220337878 & -2.1374359777 & -1.6306674943 \\ \text { H38 } & -1.7772719348 & -1.3904568789 & -2.1714898378 \\ \text { H39 } & -1.2517144728 & -1.2802236557 & -0.4388653527\end{array}$
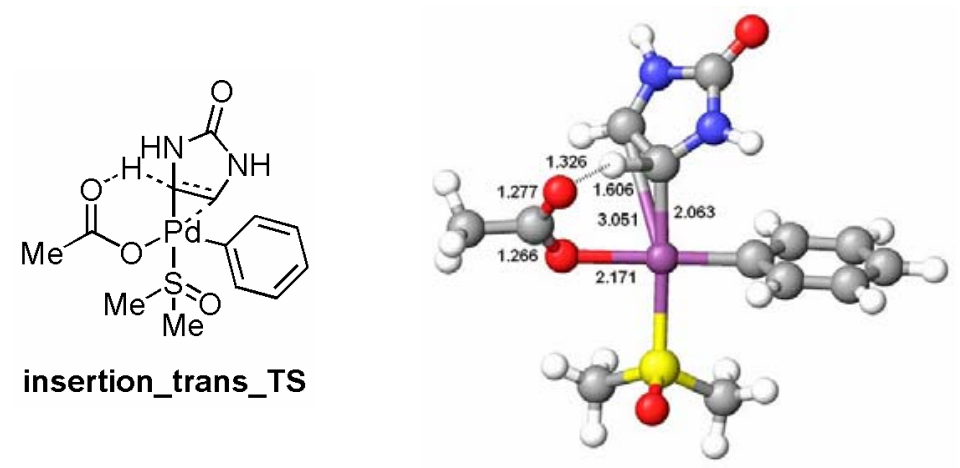

Imaginary frequency: $-1517.95 \mathrm{~cm}^{\wedge}-1$

Gas-phase SCF energy: -1441.58604450836 hartrees

Solvation energy: -0.04397794937 hartrees

Zero point energy (ZPE): $183.634 \mathrm{kcal} / \mathrm{mol}$

$\mathrm{T}=298.15 \mathrm{~K}$

Total internal energy, Utot (SCFE + ZPE + U) : -1441.267688 hartrees Total enthalpy, Htot (Utot + pV) : -1441.266744 hartrees

Total Gibbs free energy, Gtot (Htot - T*S) : -1441.348879 hartrees

Geometry Coordinates

$\begin{array}{lc}\text { atom } & \text { x } \\ \text { Pd1 } & 0.0947890000 \\ \text { C2 } & -1.6272030000 \\ \text { C3 } & -2.8722111218 \\ \text { H4 } & -3.1022448721 \\ \text { N5 } & -3.8469093651 \\ \text { H6 } & -4.8446192919 \\ \text { N7 } & -1.9509684415 \\ \text { H8 } & -1.2673646918 \\ \text { C9 } & -3.2885743335 \\ \text { O10 } & -3.8950636885 \\ \text { O11 } & -0.4387060000 \\ \text { C12 } & -0.7677330000 \\ \text { O13 } & -0.8871910000\end{array}$

$\begin{array}{cc}\begin{array}{c}\text { angstroms } \\ y\end{array} & z \\ -0.5242480001 & 0.8105439999 \\ 0.3484410000 & 0.0823320000 \\ 0.1744353405 & 0.6791067065 \\ 0.0850773325 & 1.7308173316 \\ 0.1336515612 & -0.2761533572 \\ 0.0790758825 & -0.1318326254 \\ 0.4049616455 & -1.3056338395 \\ 0.4366648174 & -2.0463045579 \\ 0.2662243250 & -1.5688179499 \\ 0.2612366544 & -2.6320811234 \\ 0.2414780000 & 2.7702150000 \\ 1.4612630000 & 2.8532190000 \\ 2.2733330000 & 1.8748620000\end{array}$




$\begin{array}{lrrr}\text { H14 } & -1.2814370000 & 1.7878660000 & 0.7059380000 \\ \text { C15 } & -0.9950235650 & 2.0578704271 & 4.2273647814 \\ \text { H16 } & -0.8140985600 & 1.3171213821 & 5.0070977137 \\ \text { H17 } & -2.0208649792 & 2.4337570975 & 4.2972133727 \\ \text { H18 } & -0.3281624907 & 2.9146575458 & 4.3629453228 \\ \text { C19 } & 0.5725860837 & -1.2536253624 & -1.0044326593 \\ \text { C20 } & 1.3560272544 & -2.3130187725 & -3.5027075522 \\ \text { C21 } & 1.6263043217 & -0.6739584804 & -1.7299052781 \\ \text { C22 } & -0.0906553260 & -2.3636588116 & -1.5532888391 \\ \text { C23 } & 0.3014659495 & -2.8927451496 & -2.7897421957 \\ \text { C24 } & 2.0144720404 & -1.2007948782 & -2.9708222986 \\ \text { H25 } & 2.1593816807 & 0.1855401943 & -1.3303111127 \\ \text { H26 } & -0.9255790356 & -2.8173290773 & -1.0251861539 \\ \text { H27 } & -0.2261226151 & -3.7510368214 & -3.1991004675 \\ \text { H28 } & 2.8327957127 & -0.7383416034 & -3.5175762981 \\ \text { H29 } & 1.6555625250 & -2.7189535016 & -4.4649643041 \\ \text { S30 } & 2.0528408887 & -1.5895960085 & 1.7858050658 \\ \text { O31 } & 3.3283800270 & -0.8006108000 & 1.9358628642 \\ \text { C32 } & 1.5638753574 & -2.1869009010 & 3.4356031472 \\ \text { H33 } & 2.4403436491 & -2.6418715144 & 3.9032512621 \\ \text { H34 } & 0.7434360499 & -2.9015726359 & 3.3339057111 \\ \text { H35 } & 1.2269483647 & -1.3133715823 & 3.9943499371 \\ \text { C36 } & 2.4726072454 & -3.1788998911 & 1.0062426198 \\ \text { H37 } & 1.5861064197 & -3.8160324440 & 0.9764799154 \\ \text { H38 } & 3.2803435766 & -3.6321364170 & 1.5856035689 \\ \text { H39 } & 2.8023831253 & -2.9548243739 & -0.0085223217\end{array}$

\section{Summary of the activation energies for trans-PdL ${ }_{2} \mathrm{X}_{2}$ : $\triangle \mathrm{SCFE}$ (gas) : $28.00 \mathrm{kcal} / \mathrm{mol}$ $\triangle \mathrm{ZPE}:-3.73 \mathrm{kcal} / \mathrm{mol}$ $\Delta \mathrm{H}($ gas $): 24.06 \mathrm{kcal} / \mathrm{mol}$ $\Delta \mathrm{G}($ gas $): 24.28 \mathrm{kcal} / \mathrm{mol}$ Solvation correction: $3.62 \mathrm{kcal} / \mathrm{mol}$}

\section{Experimental Section}

General Experimental Procedures. All reactions were performed in glassware under a positive pressure of argon. Liquids and solvent were transferred via syringe. Organic solutions were concentrated by rotary evaporator at ca. $30 \mathrm{mmHg}$. Flash column chromatography was performed as described by Still, ${ }^{\text {S6 }}$ employing EMD RP-18 silica gel 60 (230-400 mesh ASTM) or Fisher Davisil 633 silica gel (200-425 mesh). TLC analyses were performed on EMD $250 \mu \mathrm{m}$ Silica Gel $60 \mathrm{~F}_{254}$ plates and visualized by quenching of UV fluorescence $\left(\lambda_{\max }=254 \mathrm{~nm}\right)$, or by staining with phosphomolybdic acid or ceric ammonium molybdate or potassium permanganate. ${ }^{1} \mathrm{H}$ and ${ }^{13} \mathrm{C}$ NMR spectra were recorded on a Varian Inova-400 or Mercury-300 spectrometer. Chemical shifts for ${ }^{1} \mathrm{H}$ and ${ }^{13} \mathrm{C}$ NMR spectra are reported in $\mathrm{ppm}(\delta)$ relative to residue protium in the solvent $\left(\mathrm{CDCl}_{3}: \delta 7.26\right.$, $77.23 \mathrm{ppm}$; DMSO- $d_{6}: \delta 2.50,39.51 \mathrm{ppm}$ ) and the multiplicities are presented as follows: $\mathrm{s}=$ singlet, $\mathrm{d}$ $=$ doublet, $\mathrm{t}=$ triplet, $\mathrm{m}=$ multiplet. Infrared spectra were recorded on a Perkin-Elmer 1000 series FTIR. Mass spectra were acquired on a Shimadzu 2010 LC-MS using the indicated ionization method.

Materials. Commercial solvents and reagents were used as received except that dimethyl sulfoxide was degassed by freeze-pump-thaw cycles, and 2,2,2-trichloroethanol was treated with calcium sulfate and sodium bicarbonate followed by distillation. Imidazolinone $(\mathbf{1})^{\mathrm{S} 7}$ and 2-(3-bromoallyl)isoindoline-1,3dione $(\mathbf{1 1})^{\mathrm{S} 8,9}$ are both commercially available and can be easily prepared in large scales. 
General procedure for the C-arylation of imidazolinone (1). Palladium(II) acetate (6.7 mg, 0.0298 mmol, 0.05 equiv), imidazolinone (1) $(50 \mathrm{mg}, 0.595 \mathrm{mmol}, 1.0$ equiv) and sodium acetate trihydrate (242.8 mg, $1.78 \mathrm{mmol}, 3.0$ equiv) were weighed and added to an argon-filled $16 \mathrm{~mL}$ vial. Aryl iodide (0.892 mmol, 1.5 equiv) and degassed dimethyl sulfoxide $(2.5 \mathrm{~mL})$ were then added and the vial was purged with argon for $1 \mathrm{~min}$. After stirring in dark at $80^{\circ} \mathrm{C}$ with the time indicated in Table 1, the solvent was removed. The crude residue was dissolved in methanol and dry-loaded onto a RP-18 silica gel column for chromatography purification. For arylation using aryl bromides, palladium(II) acetate (13.4 mg, $0.0595 \mathrm{mmol}, 0.1$ equiv), imidazolinone (1) (50 mg, $0.595 \mathrm{mmol}, 1.0$ equiv), aryl bromide ( $0.892 \mathrm{mmol}, 1.5$ equiv, if solid) and sodium acetate trihydrate ( $242.8 \mathrm{mg}, 1.78 \mathrm{mmol}, 3.0$ equiv) were added to a $10 \mathrm{~mL}$ round-bottom flask and vigorously degassed. Degassed DMSO (2.5 mL) and aryl bromide $\left(0.892 \mathrm{mmol}, 1.5\right.$ equiv, if liquid) were then added. The reaction was stirred in dark at $80^{\circ} \mathrm{C}$ with the time indicated in Table 1 and S1.

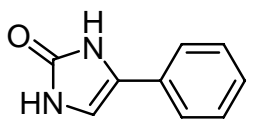

4-Phenyl-1H-imidazol-2(3H)-one. According to the general procedure using iodobenzene and purified with RP-18 silica gel column chromatography $(0 \rightarrow 80 \%$ methanol-water): $R_{f}=0.30$ ( $10 \%$ methanol-methylene chloride); FTIR (neat, $\mathrm{cm}^{-1}$ ) 1704, 1644, 1616, 1454, 1130, 938, 772, 730; ${ }^{1} \mathrm{H}$ NMR (400 MHz, DMSO-d 6$) \delta 10.50(\mathrm{~s}, 1 \mathrm{H}), 10.04(\mathrm{~s}$, $1 \mathrm{H}), 7.49(\mathrm{~d}, J=7.7 \mathrm{~Hz}, 2 \mathrm{H}), 7.31(\mathrm{t}, J=7.6 \mathrm{~Hz}, 2 \mathrm{H}), 7.16(\mathrm{t}, J=7.4 \mathrm{~Hz}, 1 \mathrm{H}), 6.88(\mathrm{~s}, 1 \mathrm{H}) ;{ }^{13} \mathrm{C} \mathrm{NMR}$ $\left(100 \mathrm{MHz}, \mathrm{DMSO}-\mathrm{d}_{6}\right) \delta 155.1,130.0,128.7,126.2,122.8,122.0,105.8 ; \mathrm{MS}\left(\mathrm{ES}^{+}\right)$calcd for $\mathrm{C}_{9} \mathrm{H}_{9} \mathrm{~N}_{2} \mathrm{O}$ $(\mathrm{M}+\mathrm{H})^{+}$161.07, found 161.00 .

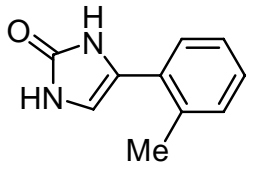

4-(2-Methylphenyl)-1H-imidazol-2(3H)-one. According to the general procedure using 2-iodotoluene and purified with RP-18 silica gel column chromatography $\left(0 \rightarrow 80 \%\right.$ methanol-water): $R_{f}=0.34$ ( $10 \%$ methanol-methylene chloride); FTIR (neat, $\left.\mathrm{cm}^{-1}\right) 1737,1559,1496,1230,1147,815,768,732 ;{ }^{1} \mathrm{H}$ NMR $\left(400 \mathrm{MHz}, \mathrm{DMSO}-d_{6}\right) \delta$ $10.28(\mathrm{~s}, 1 \mathrm{H}), 10.07(\mathrm{~s}, 1 \mathrm{H}), 7.36(\mathrm{~d}, J=7.5 \mathrm{~Hz}, 1 \mathrm{H}), 7.21(\mathrm{t}, J=7.8 \mathrm{~Hz}, 2 \mathrm{H}), 7.14(\mathrm{~d}, J=6.8 \mathrm{~Hz}, 1 \mathrm{H})$, $6.50(\mathrm{~s}, 1 \mathrm{H}), 2.34(\mathrm{~s}, 3 \mathrm{H}) ;{ }^{13} \mathrm{C}$ NMR $\left(100 \mathrm{MHz}, \mathrm{DMSO}-d_{6}\right) \delta 154.8,134.2,131.0,129.2,126.5,126.4$, 126.0, 121.1, 108.3, 21.5; $\mathrm{MS}\left(\mathrm{ES}^{+}\right)$calcd for $\mathrm{C}_{10} \mathrm{H}_{11} \mathrm{~N}_{2} \mathrm{O}(\mathrm{M}+\mathrm{H})^{+}$175.09, found 175.02.

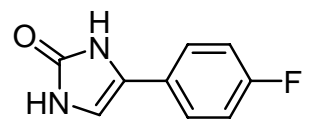

4-(4-Fluorophenyl)-1H-imidazol-2(3H)-one. According to the general procedure using 1-fluoro-4-iodobenzene and purified with $\mathrm{RP}-18$ silica gel column chromatography $(0 \rightarrow 80 \%$ methanol-water $): R_{f}=0.32(10 \%$ methanol-methylene chloride); FTIR (neat, $\left.\mathrm{cm}^{-1}\right)$ 1703, 1650, 1628, 1513, 1235, 1122, 839,739; ${ }^{1} \mathrm{H} \mathrm{NMR} \mathrm{(400} \mathrm{MHz,}$ DMSO- $\left.d_{6}\right) \delta 10.51(\mathrm{~s}, 1 \mathrm{H}), 10.04(\mathrm{~s}, 1 \mathrm{H}), 7.53(\mathrm{dd}, J=5.5 \mathrm{~Hz}, 2 \mathrm{H}), 7.17(\mathrm{t}, J=8.8 \mathrm{~Hz}, 2 \mathrm{H}), 6.86(\mathrm{~s}$, $1 \mathrm{H}) ;{ }^{13} \mathrm{C}$ NMR $\left(100 \mathrm{MHz}, \mathrm{DMSO}-d_{6}\right) \delta 160.7(\mathrm{~d}, J=242.9 \mathrm{~Hz}), 155.1,126.7(\mathrm{~d}, J=30.0 \mathrm{~Hz}), 124.7(\mathrm{~d}$, $J=7.6 \mathrm{~Hz}), 121.2,115.6(\mathrm{~d}, J=31.8 \mathrm{~Hz}), 105.7$; $\mathrm{MS}\left(\mathrm{ES}^{+}\right)$calcd for $\mathrm{C}_{9} \mathrm{H}_{8} \mathrm{FN}_{2} \mathrm{O}(\mathrm{M}+\mathrm{H})^{+} 179.06$, found 179.00 .

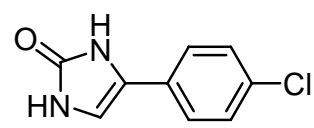

4-(4-Chlorophenyl)-1H-imidazol-2(3H)-one. According to the general procedure using 1-chloro-4-iodobenzene and purified with RP-18 silica gel column chromatography $(0 \rightarrow 80 \%$ methanol-water $): R_{f}=0.35$ (10\% methanol-methylene chloride); FTIR (neat, $\mathrm{cm}^{-1}$ ) 1736, 1654, 1622, 1498, 1420, 1133, 1097, 826, 730; ${ }^{1} \mathrm{H} \mathrm{NMR} \mathrm{(400} \mathrm{MHz,}$ DMSO- $\left.d_{6}\right) \delta 10.55(\mathrm{~s}, 1 \mathrm{H}), 10.12(\mathrm{~s}, 1 \mathrm{H}), 7.51(\mathrm{~d}, J=8.6 \mathrm{~Hz}, 2 \mathrm{H}), 7.38(\mathrm{~d}, J=8.6 \mathrm{~Hz}, 2 \mathrm{H}), 6.96(\mathrm{~s}$, $1 \mathrm{H}) ;{ }^{13} \mathrm{C}$ NMR $\left(100 \mathrm{MHz}, \mathrm{DMSO}-d_{6}\right) \oint 155.0,130.3,129.0,128.7,124.4,106.7$; $\mathrm{MS}\left(\mathrm{ES}^{+}\right)$calcd for $\mathrm{C}_{9} \mathrm{H}_{8} \mathrm{ClN}_{2} \mathrm{O}(\mathrm{M}+\mathrm{H})^{+}$195.03, found 194.95.

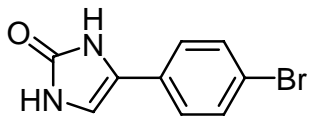

4-(4-Bromophenyl)-1H-imidazol-2(3H)-one. According to the general procedure using 1-bromo-4-iodobenzene and purified with RP-18 silica gel column 
chromatography $(0 \rightarrow 80 \% \mathrm{~N}, \mathrm{~N}$-dimethylformamide-water $): R_{f}=0.36(10 \%$ methanol-methylene chloride); FTIR (neat, $\mathrm{cm}^{-1}$ ) 1759, 1735, 1621, 1490, 1033, 1010, 826, 782, 739; ${ }^{1} \mathrm{H} \mathrm{NMR} \mathrm{(400} \mathrm{MHz,}$ DMSO- $\left.d_{6}\right) \delta 10.55(\mathrm{~s}, 1 \mathrm{H}), 10.13(\mathrm{~s}, 1 \mathrm{H}), 7.51(\mathrm{~d}, J=8.5 \mathrm{~Hz}, 2 \mathrm{H}), 7.45(\mathrm{~d}, J=8.5 \mathrm{~Hz}, 2 \mathrm{H}), 6.79(\mathrm{~s}$, $1 \mathrm{H}) ;{ }^{13} \mathrm{C}$ NMR $\left.\left(100 \mathrm{MHz}, \mathrm{DMSO}-d_{6}\right) \delta 155.0,131.5,129.3,124.7,120.9,118.7,106.8 ; \mathrm{MS} \mathrm{ES}^{+}\right) \mathrm{calcd}^{+}$ for $\mathrm{C}_{9} \mathrm{H}_{8} \mathrm{BrN}_{2} \mathrm{O}(\mathrm{M}+\mathrm{H})^{+}$238.98, found 238.90 .

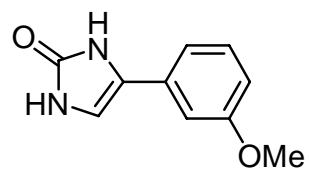

4-(3-Methoxyphenyl)-1H-imidazol-2(3H)-one. According to the general procedure using 3-iodoanisole and purified with RP-18 silica gel column chromatography $(0 \rightarrow 80 \%$ methanol-water $): R_{f}=0.34(10 \%$ methanol-methylene chloride); FTIR (neat, $\mathrm{cm}^{-1}$ ) 1718, 1618, 1577, 1221, 1046, 864, 781, 744; ${ }^{1} \mathrm{H}$ NMR (400 MHz, DMSO-d $) \delta 10.49(\mathrm{~s}, 1 \mathrm{H}), 10.05(\mathrm{~s}, 1 \mathrm{H}), 7.22$ (t, $J=7.6 \mathrm{~Hz}, 1 \mathrm{H}), 7.09$ (s, 2H), 6.91 $(\mathrm{s}, 1 \mathrm{H}), 6.73(\mathrm{~d}, J=8.1 \mathrm{~Hz}, 1 \mathrm{H}), 3.75(\mathrm{~s}, 3 \mathrm{H}) ;{ }^{13} \mathrm{C}$ NMR $\left(100 \mathrm{MHz}, \mathrm{DMSO}-d_{6}\right) \delta 159.7,155.0,131.3$, 129.7, 121.9, 115.3, 111.9, 108.4, 106.2, 55.1; $\mathrm{MS}\left(\mathrm{ES}^{+}\right)$calcd for $\mathrm{C}_{10} \mathrm{H}_{11} \mathrm{~N}_{2} \mathrm{O}_{2}(\mathrm{M}+\mathrm{H})^{+} 191.08$, found 191.00 .

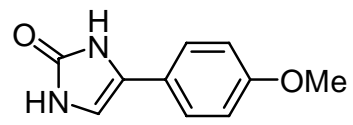

4-(4-Methoxyphenyl)-1H-imidazol-2(3H)-one. According to the general procedure using 4-iodoanisole and purified with $\mathrm{RP}-18$ silica gel column chromatography $(0 \rightarrow 80 \% \mathrm{~N}, N$-dimethylformamide -water $): R_{f}=0.34(10 \%$ methanol-methylene chloride); FTIR (neat, $\mathrm{cm}^{-1}$ ) 1700, 1607, 1516, 1252, 1182, 1032, 839, 744, 723; ${ }^{1} \mathrm{H}$ NMR $\left(400 \mathrm{MHz}, \mathrm{DMSO}-d_{6}\right) \delta 10.40(\mathrm{~s}, 1 \mathrm{H}), 9.90(\mathrm{~s}, 1 \mathrm{H}), 7.43(\mathrm{~d}, J=8.7 \mathrm{~Hz}, 2 \mathrm{H}), 6.89(\mathrm{~d}, J=8.7$ $\mathrm{Hz}, 2 \mathrm{H}), 6.71(\mathrm{~s}, 1 \mathrm{H}), 3.74(\mathrm{~s}, 3 \mathrm{H}) ;{ }^{13} \mathrm{C}$ NMR $\left(100 \mathrm{MHz}, \mathrm{DMSO}-d_{6}\right) \delta 157.8,155.0,124.2,122.8$, 121.9, 114.2, 104.0, 55.1; $\mathrm{MS}\left(\mathrm{ES}^{+}\right)$calcd for $\mathrm{C}_{10} \mathrm{H}_{11} \mathrm{~N}_{2} \mathrm{O}_{2}(\mathrm{M}+\mathrm{H})^{+}$191.08, found 191.05.

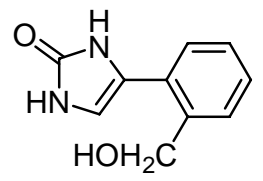

4-(2-(Hydroxymethyl)phenyl)-1H-imidazol-2(3H)-one. According to the general procedure using 2-iodobenzyl alcohol and purified with RP-18 silica gel column chromatography $(0 \rightarrow 80 \%$ methanol-water $): R_{f}=0.17$ (10\% methanol-methylene chloride); FTIR (neat, $\mathrm{cm}^{-1}$ ) 1739, 1707, 1618, 1450, 1230, 1042, 810, 768, $735 ;{ }^{1} \mathrm{H}$ NMR (400 MHz, DMSO-d $\left.d_{6}\right) \delta 10.26(\mathrm{~s}, 1 \mathrm{H}), 10.08(\mathrm{~s}, 1 \mathrm{H}), 7.48(\mathrm{~d}, J=7.0 \mathrm{~Hz}, 1 \mathrm{H}), 7.39(\mathrm{~d}, J=7.3$ $\mathrm{Hz}, 1 \mathrm{H}), 7.29-7.22(\mathrm{~m}, 2 \mathrm{H}), 6.62(\mathrm{~s}, 1 \mathrm{H}), 5.35(\mathrm{~s}, 1 \mathrm{H}), 4.50(\mathrm{~s}, 2 \mathrm{H}) ;{ }^{13} \mathrm{C}$ NMR $\left(100 \mathrm{MHz}, \mathrm{DMSO}-d_{6}\right) \delta$ $154.8,138.0,128.7,128.5,127.2,126.40,126.35,120.1,108.5,61.7 ; \mathrm{MS}\left(\mathrm{ES}^{+}\right)$calcd for $\mathrm{C}_{10} \mathrm{H}_{10} \mathrm{~N}_{2} \mathrm{NaO}_{2}(\mathrm{M}+\mathrm{Na})^{+} 213.06$, found 213.00 .

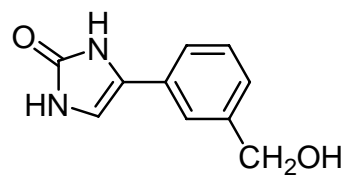

4-(3-(Hydroxymethyl)phenyl)-1H-imidazol-2(3H)-one. According to the general procedure using 3-iodobenzyl alcohol and purified with RP-18 silica gel column chromatography $(0 \rightarrow 80 \%$ methanol-water $): R_{f}=0.09(10 \%$ methanolmethylene chloride); FTIR (neat, $\mathrm{cm}^{-1}$ ) 1679, 1619, 1227, 1195, 1017, 791,739; ${ }^{1} \mathrm{H}$ NMR $\left(400 \mathrm{MHz}, \mathrm{DMSO}-d_{6}\right) \delta 10.50(\mathrm{~s}, 1 \mathrm{H}), 10.04(\mathrm{~s}, 1 \mathrm{H}), 7.45(\mathrm{~s}, 1 \mathrm{H}), 7.36(\mathrm{~d}, J=7.7 \mathrm{~Hz}, 1 \mathrm{H})$, $7.27(\mathrm{t}, J=7.5 \mathrm{~Hz}, 1 \mathrm{H}), 7.13(\mathrm{~d}, J=7.5 \mathrm{~Hz}, 1 \mathrm{H}), 6.85(\mathrm{~s}, 1 \mathrm{H}), 5.19(\mathrm{~s}, 1 \mathrm{H}), 4.46(\mathrm{~s}, 2 \mathrm{H}) ;{ }^{13} \mathrm{C} \mathrm{NMR}$ $\left.\left(100 \mathrm{MHz}, \mathrm{DMSO}-d_{6}\right) \delta 155.0,142.9,129.7,128.5,124.5,122.1,121.2,121.0,105.6,63.0 ; \mathrm{MS}_{(\mathrm{ES}}{ }^{+}\right)$ calcd for $\mathrm{C}_{10} \mathrm{H}_{11} \mathrm{~N}_{2} \mathrm{O}_{2}(\mathrm{M}+\mathrm{H})^{+}$191.08, found 191.05.

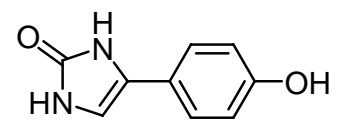

4-(4-Hydroxyphenyl)-1H-imidazol-2(3H)-one. According to the general procedure using 4-iodophenol and purified with RP-18 silica gel column chromatography $(0 \rightarrow 80 \%$ methanol-water $): R_{f}=0.10$ ( $10 \%$ methanol-methylene chloride); FTIR (neat, $\mathrm{cm}^{-1}$ ) 1679, 1602, 1514, 1250, 1179, 1100, 834, 762, 736; ${ }^{1} \mathrm{H} \mathrm{NMR} \mathrm{(400} \mathrm{MHz,}$ DMSO- $\left.d_{6}\right) \delta 10.38(\mathrm{~s}, 1 \mathrm{H}), 9.84(\mathrm{~s}, 1 \mathrm{H}), 9.46(\mathrm{~s}, 1 \mathrm{H}), 7.30(\mathrm{~d}, J=8.6 \mathrm{~Hz}, 2 \mathrm{H}), 6.71(\mathrm{~d}, J=8.6 \mathrm{~Hz}, 2 \mathrm{H})$, $6.60(\mathrm{~s}, 1 \mathrm{H}) ;{ }^{13} \mathrm{C}$ NMR $\left(100 \mathrm{MHz}, \mathrm{DMSO}-d_{6}\right) \delta 156.1,155.1,124.4,122.4,121.1,115.5,103.2$; $\mathrm{MS}\left(\mathrm{ES}^{+}\right)$calcd for $\mathrm{C}_{9} \mathrm{H}_{9} \mathrm{~N}_{2} \mathrm{O}_{2}(\mathrm{M}+\mathrm{H})^{+}$177.07, found 177.00 . 


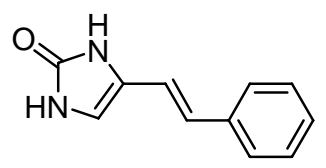

(E)-4-Styryl-1H-imidazol-2(3H)-one. According to the general procedure using $\beta$-bromostyrene and purified with RP-18 silica gel column chromatography $\left(0 \rightarrow 80 \% N, N\right.$-dimethylformamide-water): $R_{f}=0.20$ ( $10 \%$ methanol-methylene chloride); FTIR (neat, $\mathrm{cm}^{-1}$ ) 1702, 1641, 1460, 1451, 1113, 949; ${ }^{1} \mathrm{H}$ NMR $(400$ MHz, DMSO- $\left.d_{6}\right) \delta 10.35(\mathrm{~s}, 1 \mathrm{H}), 10.01(\mathrm{~s}, 1 \mathrm{H}), 7.35-7.14(\mathrm{~m}, 4 \mathrm{H}), 6.71(\mathrm{~d}, J=6.3 \mathrm{~Hz}, 1 \mathrm{H}), 6.65(\mathrm{~d}, J$ $=6.3 \mathrm{~Hz}, 1 \mathrm{H}), 6.47(\mathrm{~s}, 1 \mathrm{H}) ;{ }^{13} \mathrm{C}$ NMR $\left(100 \mathrm{MHz}, \mathrm{DMSO}-d_{6}\right) \delta 155.5,137.9,129.4,127.5,126.3,123.8$, 122.6, 117.1, 110.9; $\mathrm{MS}\left(\mathrm{ES}^{+}\right)$calcd for $\mathrm{C}_{11} \mathrm{H}_{11} \mathrm{~N}_{2} \mathrm{O}(\mathrm{M}+\mathrm{H})^{+}$187.09, found 187.00.

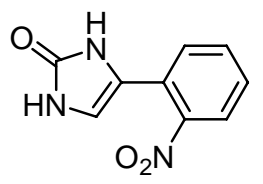

4-(2-Nitrophenyl)-1H-imidazol-2(3H)-one. According to the general procedure using 1-bromo-2-nitrobenzene and purified with RP-18 silica gel (RP-18) column chromatography $\left(0 \rightarrow 80 \% N, N\right.$-dimethylformamide -water): $R_{f}=0.35$ ( $10 \%$ methanolmethylene chloride); FTIR (neat, $\mathrm{cm}^{-1}$ ) 1995, 1705, 1614, 1520, 1354, 1027, 765, 734; ${ }^{1} \mathrm{H}$ NMR $\left(400 \mathrm{MHz}, \mathrm{DMSO}-d_{6}\right) \delta 10.34(\mathrm{~s}, 1 \mathrm{H}), 10.21(\mathrm{~s}, 1 \mathrm{H}), 7.89(\mathrm{~d}, J=8.0 \mathrm{~Hz}, 1 \mathrm{H}), 7.66(\mathrm{~d}, J=8.0$ $\mathrm{Hz}, 1 \mathrm{H}), 7.55(\mathrm{~d}, J=7.9 \mathrm{~Hz}, 1 \mathrm{H}), 7.49-7.45(\mathrm{~m}, 1 \mathrm{H}), 6.50(\mathrm{~s}, 1 \mathrm{H}) ;{ }^{13} \mathrm{C}$ NMR $\left(100 \mathrm{MHz}\right.$, DMSO- $\left.d_{6}\right) \delta$ $155.3,147.6,133.5,130.5,128.9,124.9,124.2,117.8,109.4 ; \mathrm{MS}\left(\mathrm{ES}^{+}\right)$calcd for $\mathrm{C}_{9} \mathrm{H}_{8} \mathrm{~N}_{3} \mathrm{O}_{3}(\mathrm{M}+\mathrm{H})^{+}$ 206.06, found 206.00 .

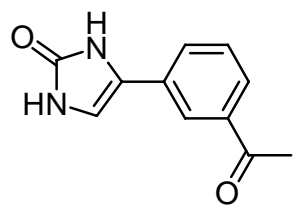

4-(3-Acetylphenyl)-1H-imidazol-2(3H)-one. According to the general procedure using 3-bromoactophenone and purified with RP-18 silica gel column chromatography $\left(0 \rightarrow 80 \% N, N\right.$-dimethylformamide-water): $R_{f}=0.25(10 \%$ methanol-methylene chloride); FTIR (neat, $\mathrm{cm}^{-1}$ ) 1995, 1690, 1620, 1462, 1427, 1253,$738 ;{ }^{1} \mathrm{H}$ NMR $\left(400 \mathrm{MHz}, \mathrm{DMSO}-d_{6}\right) \delta 10.64(\mathrm{~s}, 1 \mathrm{H}), 10.13(\mathrm{~s}, 1 \mathrm{H}), 8.05(\mathrm{~s}$, $1 \mathrm{H}), 7.73-7.67(\mathrm{~m}, 2 \mathrm{H}), 7.44(\mathrm{t}, \mathrm{d}, J=7.7 \mathrm{~Hz}, 1 \mathrm{H}) 7.04(\mathrm{~s}, 1 \mathrm{H}), 2.57(\mathrm{~s}, 3 \mathrm{H}) ;{ }^{13} \mathrm{C}$ NMR $(100 \mathrm{MHz}$, DMSO- $\left.d_{6}\right) \delta 203.4,160.4,142.7,135.9,134.4,132.5,130.8,128.0,126.5,32.3 ;$ MS(ES ${ }^{+}$) calcd for $\mathrm{C}_{11} \mathrm{H}_{11} \mathrm{~N}_{2} \mathrm{O}_{2}(\mathrm{M}+\mathrm{H})^{+}$203.08, found 203.00.

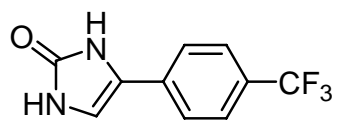

4-(4-(Trifluoromethyl)phenyl)-1H-imidazol-2(3H)-one. According to the general procedure using 4-bromobenzotrifluoride and purified with RP-18 silica gel column chromatography $(0 \rightarrow 80 \% N, N$-dimethylformamide-water $): R_{f}=0.20$ (10\% methanol-methylene chloride); FTIR (neat, $\left.\mathrm{cm}^{-1}\right)$ 1758, 1707, 1618, 1450, 1333, 1132, 1063, 844, 747, 723; ${ }^{1} \mathrm{H}$ NMR $\left(400 \mathrm{MHz}\right.$, DMSO- $\left.d_{6}\right) \delta 10.66(\mathrm{~s}, 1 \mathrm{H}), 10.25(\mathrm{~s}, 1 \mathrm{H}), 7.68-7.62(\mathrm{~m}, 4 \mathrm{H}), 7.12(\mathrm{~s}$, $1 \mathrm{H}) ;{ }^{13} \mathrm{C}$ NMR $\left(100 \mathrm{MHz}\right.$, DMSO- $\left.d_{6}\right) \delta 154.9,133.9,126.0(\mathrm{q}, J=31.7 \mathrm{~Hz}), 125.6(\mathrm{q}, J=3.4 \mathrm{~Hz})$, $124.4(\mathrm{q}, J=270.1 \mathrm{~Hz}), 120.7,118.4,108.6$; $\mathrm{MS}\left(\mathrm{ES}^{+}\right)$calcd for $\mathrm{C}_{10} \mathrm{H}_{8} \mathrm{~F}_{3} \mathrm{~N}_{2} \mathrm{O}(\mathrm{M}+\mathrm{H})^{+} 229.06$, found 228.95 .

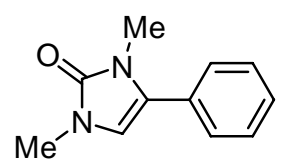

1,3-Dimethyl-4-phenyl-1H-imidazol-2(3H)-one (6). According to the general procedure using 5 and iodobenzene and purified with silica gel (Davisil 633) column chromatography: $R_{f}=0.11$ (100\% ethyl acetate); FTIR (neat, $\left.\mathrm{cm}^{-1}\right) 2925,1715,1681$, 1467, 1398, 1291, 1183, 1041, 1016, 855, 787, 766, 728; ${ }^{1} \mathrm{H} \mathrm{NMR}\left(400 \mathrm{MHz}, \mathrm{CDCl}_{3}\right.$ ) $\delta$ 7.40-7.29 (m, 5H), $6.21(\mathrm{~s}, 1 \mathrm{H}), 3.30(\mathrm{~s}, 3 \mathrm{H}), 3.27(\mathrm{~s}, 3 \mathrm{H}) ;{ }^{13} \mathrm{C} \mathrm{NMR}\left(75 \mathrm{MHz}, \mathrm{CDCl}_{3}\right) \delta 154.3$, 129.8, 129.0, 128.2, 127.7, 125.0, 109.1, 30.6, 29.5; $\mathrm{MS}\left(\mathrm{ES}^{+}\right)$calcd for $\mathrm{C}_{11} \mathrm{H}_{13} \mathrm{~N}_{2} \mathrm{O}(\mathrm{M}+\mathrm{H})^{+} 189.10$, found 189.05 .

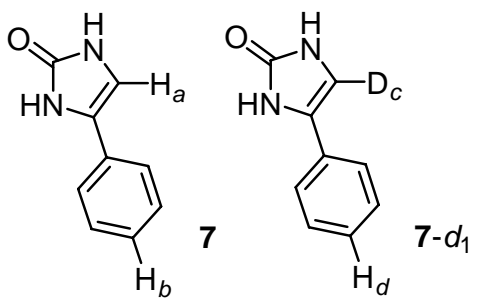

Kinetic isotopic effect experiment. According to the general procedure, 1 (5.0 mg, $0.06 \mathrm{mmol}, 1.0$ equiv), $1-d_{2}(5.0 \mathrm{mg}, 0.06 \mathrm{mmol}, 1.0$ equiv, containing $12 \%$ imidzol-2-one- $\left.d_{1}\right)$, iodobenzene $(0.67 \mu \mathrm{L}, 0.006 \mathrm{mmol}$, 0.1 equiv), palladium(II) acetate ( $1.3 \mathrm{mg}, 0.006 \mathrm{mmol}, 0.2$ equiv), and sodium acetate trihydrate $(50 \mathrm{mg}, 0.36 \mathrm{mmol}, 6.0$ equiv) was stirred in 
$\operatorname{DMSO}(0.5 \mathrm{~mL})$ at $80{ }^{\circ} \mathrm{C}$ for $12 \mathrm{~h}$. The ratio of 7 and $7-d_{1}$ was determined to be $4: 1$ by comparing the integration of the vinyl $\mathrm{C}-\mathrm{H}\left(\mathrm{H}_{a}\right)$ and aryl $\mathrm{C}-\mathrm{H}\left(\mathrm{H}_{b, d}\right)$ in the ${ }^{1} \mathrm{H}$ NMR spectra. A 10 s recycle delay was used and the integration was calibrated with the pure sample of 7. A kinetic isotope effect of $k_{\mathrm{H}} / k_{\mathrm{D}} \approx$ 4.55 was derived from the following equations: $\left(\mathrm{H}_{b}+\mathrm{H}_{d}\right) / \mathrm{H}_{a}=1.25: 1 \Rightarrow 7: 7-d_{1}=4: 1 ;\left(\left(0.50 \times 2 k_{\mathrm{H}}+\right.\right.$ $\left.\left.0.06 k_{\mathrm{D}}\right) /\left(0.06 k_{\mathrm{H}}+0.44 \times 2 k_{\mathrm{D}}\right)\right)=4: 1 \Rightarrow k_{\mathrm{H}} / k_{\mathrm{D}}=4.55$.<smiles>Cn1cccc1</smiles>

1-Difluoromethyl-3-methyl-1H-imidazol-2-one (8). A modified procedure was used to form the imidazol-2-one preferentially under aerobic conditions. ${ }^{\mathrm{S} 10} \mathrm{~A}$ mixture of $N$-methyl-imidazole (328.5 mg, $4.0 \mathrm{mmol}, 1.0$ equiv) and sodium fluoride ( $16.8 \mathrm{mg}, 0.4 \mathrm{mmol}, 0.1$ equiv) in anhydrous 1,2 -dimethoxyethane $(20 \mathrm{~mL})$ was stirred at $85^{\circ} \mathrm{C}$ for 20 minutes before trimethylsilylfluorosulfonyldifluoroacetate ( $4.00 \mathrm{~g}, 16.0 \mathrm{mmol}, 4.0$ equiv) was added dropwise. After stirring the reaction at $85^{\circ} \mathrm{C}$ for $16 \mathrm{~h}$, the solvent was removed and the residue was purified by silica gel flash chromatography to give $\mathbf{8}$ as yellow oil (202.0 mg, 34\%): $R_{f}=0.22$ (50\% ethyl acetate-hexanes); FTIR (neat, $\mathrm{cm}^{-1}$ ) 1718, 1560, 1443, 1396, 1131; ${ }^{1} \mathrm{H}$ NMR (400 MHz, $\left.\mathrm{CDCl}_{3}\right) \delta 7.04(\mathrm{t}, J=59.9 \mathrm{~Hz}, 1 \mathrm{H}), 6.47(\mathrm{~d}, J=3.2 \mathrm{~Hz}, 1 \mathrm{H})$, $6.26(\mathrm{~d}, J=2.9 \mathrm{~Hz}, 1 \mathrm{H}), 3.24(\mathrm{~s}, 3 \mathrm{H}) ;{ }^{13} \mathrm{C} \mathrm{NMR}\left(75 \mathrm{MHz}, \mathrm{CDCl}_{3}\right) \delta 158.8,114.9,107.6(\mathrm{t}, J=244.0$ $\mathrm{Hz}), 104.5,30.2$; $\mathrm{MS}\left(\mathrm{ES}^{+}\right)$calcd for $\mathrm{C}_{5} \mathrm{H}_{6} \mathrm{~F}_{2} \mathrm{~N}_{2} \mathrm{O}(\mathrm{M}+\mathrm{H})^{+}$149.04, found 149.00 .<smiles>[2H]c1cn(C(F)F)c(=O)n1C</smiles>

4-Deutero-1-difluoromethyl-3-methyl-1H-imidazol-2(3H)-one (8- $\left.\boldsymbol{d}_{1}\right)$. To a solution of 2,5-diiodo-1-methylimidazole ${ }^{\mathrm{S} 11}$ (1.00 g, $3.0 \mathrm{mmol}, 1.0$ equiv) in tetrahydrofuran-ether $(1: 1,10 \mathrm{~mL})$ at $-78^{\circ} \mathrm{C}$ was added tert-butyllithium $(1.7 \mathrm{M}$ in pentane, $7.06 \mathrm{~mL}, 12.0 \mathrm{mmol}, 4.0$ equiv) dropwise along the flask wall. After stirring for $5 \mathrm{~min}$, deuterium oxide $(1.0 \mathrm{~mL})$ was added. The aqueous phase was removed and the organic phase was concentrated. The residue was re-dissolved in methylene chloride, dried with anhydrous sodium sulfate, filtrated and concentrated to give the 2,5-dideutero-1methylimidazole as colorless oil, which was converted to 8- $\boldsymbol{d}_{\boldsymbol{1}}$ as described above $(93 \% \mathrm{D}$ at C-4 and $9 \% \mathrm{D}$ at $\mathrm{C}-5$ by NMR).

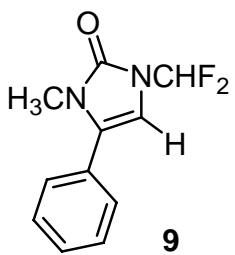

9

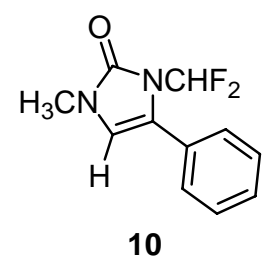

10

1-Difluoromethyl-3-methyl-4-phenyl-1 $H$-imidazol-2(3H)-one (9) and 1-difluoromethyl-3-methyl-5-phenyl-1 $\mathrm{H}$-imidazol-2(3H)-one (10). According to the general procedure, imidazolinone 8 was reacted with iodobenzene at $23{ }^{\circ} \mathrm{C}$ for $48 \mathrm{~h}$ to give $\mathbf{9}$ and $\mathbf{1 0}(6: 1)$ as determined by NMR with $10 \mathrm{~s}$ recycle delay. Imidazolinone $\mathbf{9}$ and $\mathbf{1 0}$ can be separated by PTLC $\left(250 \mu \mathrm{m}\right.$ Silica Gel $\left.60 \mathrm{~F}_{254}\right)$. 9: $R_{f}=0.44$ (50\% ethyl acetate-hexanes); FT-IR (neat, $\mathrm{cm}^{-1}$ ) 1726, 1710, 1459, 1396, 1238, 1180, 1172, 1112, 1022, 921, 893, 770; ${ }^{1} \mathrm{H}$ NMR $\left(400 \mathrm{MHz}, \mathrm{CDCl}_{3}\right) \delta 7.49-7.35(\mathrm{~m}, 5 \mathrm{H}), 7.15(\mathrm{t}, J=60.0 \mathrm{~Hz}), 6.49(\mathrm{~s}, 1 \mathrm{H})$, $3.25(\mathrm{~s}, 3 \mathrm{H}) ;{ }^{13} \mathrm{C} \mathrm{NMR}\left(100 \mathrm{MHz}, \mathrm{CDCl}_{3}\right) \delta 152.3,129.3,129.2,128.5,128.4,128.1,107.7(\mathrm{t}, J=$ 244.1 Hz), 101.6, 29.0; $\mathrm{MS}\left(\mathrm{ES}^{+}\right)$calcd for $\mathrm{C}_{11} \mathrm{H}_{10} \mathrm{~F}_{2} \mathrm{~N}_{2} \mathrm{O}(\mathrm{M}+\mathrm{H})^{+}$225.08, found 224.95. 10: $R_{f}=0.26$ (50\% ethyl acetate-hexanes); FTIR (neat, $\mathrm{cm}^{-1}$ ) 1709, 1437, 1453, 1401, 1355, 1262, 1121, 1047, 1006, 891, 804, 762; ${ }^{1} \mathrm{H}$ NMR (400 MHz, $\left.\mathrm{CDCl}_{3}\right) \delta 7.48-7.37(\mathrm{~m}, 5 \mathrm{H}), 7.20(\mathrm{t}, J=59.2 \mathrm{~Hz}, 1 \mathrm{H}), 6.24(\mathrm{~s}, 1 \mathrm{H})$, $3.31(\mathrm{~s}, 3 \mathrm{H}) ;{ }^{13} \mathrm{C}$ NMR $\left(100 \mathrm{MHz}, \mathrm{CDCl}_{3}\right) \delta 152.6,128.8,128.7,128.6,122.1,112.5,109.4(\mathrm{t}, J=$ $245.8 \mathrm{~Hz}), 30.4$; $\mathrm{MS}\left(\mathrm{ES}^{+}\right)$calcd for $\mathrm{C}_{11} \mathrm{H}_{10} \mathrm{~F}_{2} \mathrm{~N}_{2} \mathrm{O}(\mathrm{M}+\mathrm{H})^{+}$225.08, found 224.95.

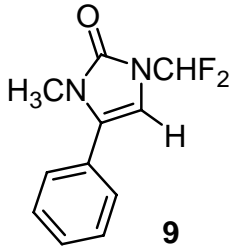<smiles>[2H]c1[nH]c(=O)n(Cc2ccccc2)c1-c1ccccc1</smiles>

1-Difluoromethyl-3-methyl-4-phenyl-1H-imidazol-2(3H)-one (9) and 4-Deutero-1-difluoromethyl-3-methyl-5-phenyl-1 $H$-imidazol2(3H)-one (10- $\left.\boldsymbol{d}_{1}\right)$. According to the procedure described above, 8- $\boldsymbol{d}_{\boldsymbol{1}}$ and iodobenzene were reacted at $28{ }^{\circ} \mathrm{C}$ for $33 \mathrm{~h}$ to give $9(10 \% \mathrm{D})$ and 10- $\boldsymbol{d}_{\boldsymbol{1}}(87 \% \mathrm{D})$ after purified by PTLC $(250 \mu \mathrm{m}$ Silica Gel 60 $\left.\mathrm{F}_{254}\right)$. 

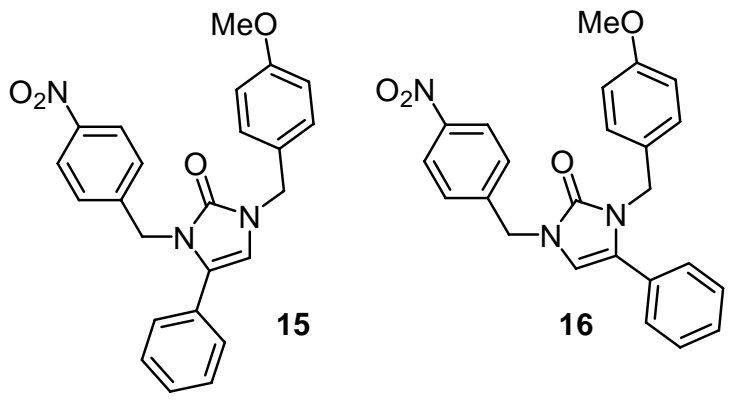

1-(4-Methoxybenzyl)-3-(4-nitrobenzyl)-4-phenyl-1Himidazol-2(3H)-one (15) and 1-(4-methoxybenzyl)-3(4-nitrobenzyl)-5-phenyl-1H-imidazol-2(3H)-one (16). According to the general procedure using imidazolinone 13 and iodobenzene, $\mathbf{1 4}$ and $\mathbf{1 5}$ were obtained as yellow oil from PTLC $\left(250 \mu \mathrm{m}\right.$ Silica Gel $\left.60 \mathrm{~F}_{254}\right)$ in 1.8:1 ratio (ca. 20\% conversion by ${ }^{1} \mathrm{H}$ NMR). 14: $R_{f}=0.35(50 \%$ ethyl acetate-hexanes); FT-IR (neat, $\mathrm{cm}^{-1}$ ) 1682, 1608, $1514,1453,1409,1344,1247,1176,1110,1032,819$,

764, 734; ${ }^{1} \mathrm{H}$ NMR $\left(400 \mathrm{MHz}, \mathrm{CDCl}_{3}\right) \delta 8.11(\mathrm{~d}, J=8.8 \mathrm{~Hz}, 2 \mathrm{H}), 7.30-7.25(\mathrm{~m}, 7 \mathrm{H}), 7.12-7.09(\mathrm{~m}, 2 \mathrm{H})$, $6.89(\mathrm{~d}, J=8.8 \mathrm{~Hz}, 2 \mathrm{H}) ;{ }^{13} \mathrm{C} \mathrm{NMR}\left(75 \mathrm{MHz}, \mathrm{CDCl}_{3}\right) \delta 159.6,147.5,145.4,129.8,129.3,129.1,129.0$, $128.8,128.7,128.6,128.3,128.1,127.8,124.1,114.5,108.7,55.6,47.2,45.2$; MS(ES ${ }^{+}$) calcd for $\mathrm{C}_{24} \mathrm{H}_{22} \mathrm{~N}_{3} \mathrm{O}_{4}(\mathrm{M}+\mathrm{H})^{+} 416.16$, found 416.10. 15: $R_{f}=0.33$ (50\% ethyl acetate-hexanes); FTIR (neat, $\mathrm{cm}^{-}$ $\left.{ }^{1}\right) 1682,1609,1514,1453,1410,1284,1176,1108,1032,846,801,767,733 ;{ }^{1} \mathrm{H}$ NMR (400 MHz, $\left.\mathrm{CDCl}_{3}\right) \delta 8.21(\mathrm{~d}, J=8.4 \mathrm{~Hz}, 2 \mathrm{H}), 7.46(\mathrm{~d}, J=8.8 \mathrm{~Hz}, 2 \mathrm{H}), 7.34-7.32(\mathrm{~m}, 3 \mathrm{H}), 7.21-7.19(\mathrm{~m}, 2 \mathrm{H}), 7.00$ $(\mathrm{d}, J=8.8 \mathrm{~Hz}, 2 \mathrm{H}), 6.76(\mathrm{~d}, J=8.6 \mathrm{~Hz}, 2 \mathrm{H}), 6.17(\mathrm{~s}, 1 \mathrm{H}), 4.98(\mathrm{~s}, 2 \mathrm{H}), 4.87(\mathrm{~s}, 2 \mathrm{H}), 3.76(\mathrm{~s}, 3 \mathrm{H}) ;{ }^{13} \mathrm{C}$ NMR $\left(75 \mathrm{MHz}, \mathrm{CDCl}_{3}\right) \delta 159.1,154.2,147.8,144.6,129.9,129.4,128.9,128.8,128.7,128.6,126.0$, 124.3, 114.1, 107.9, 55.5, 46.9, 45.3; $\mathrm{MS}\left(\mathrm{ES}^{+}\right)$calcd for $\mathrm{C}_{24} \mathrm{H}_{22} \mathrm{~N}_{3} \mathrm{O}_{4}(\mathrm{M}+\mathrm{H})^{+}$416.16, found 416.10.

Scheme S6.
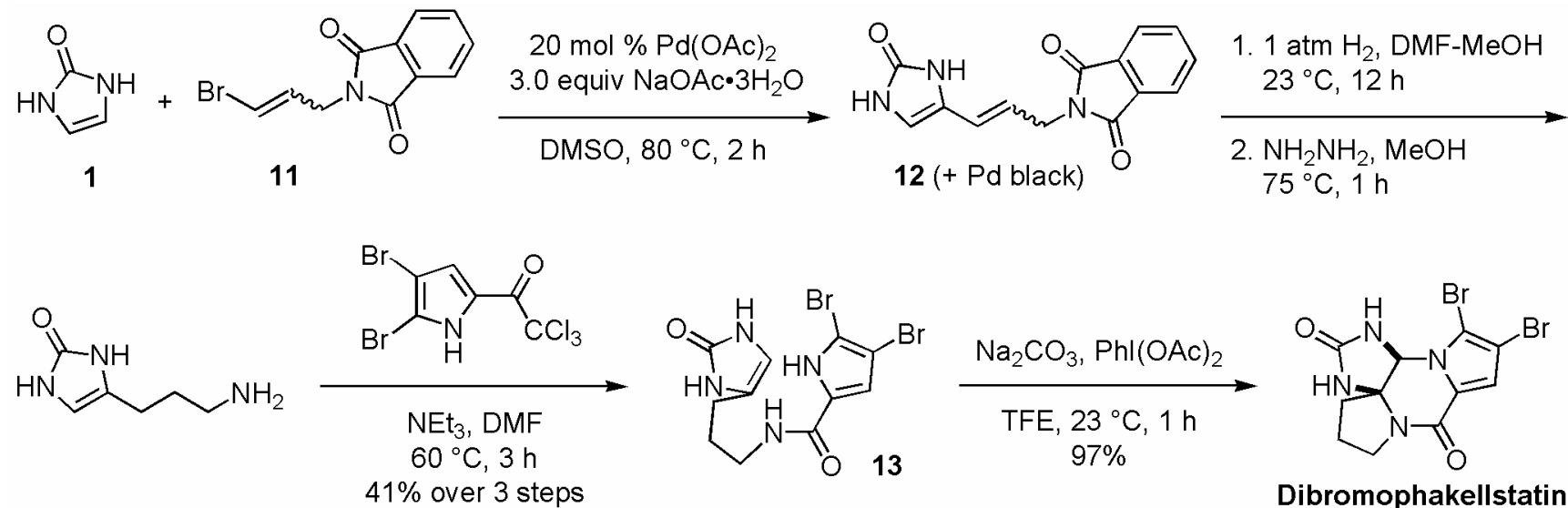

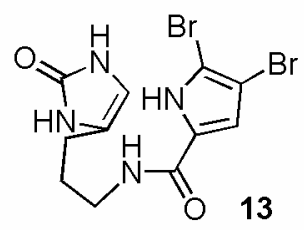

13

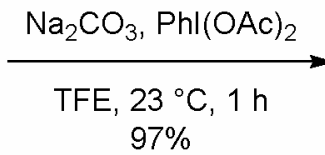
$97 \%$

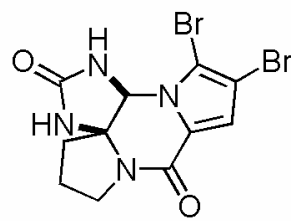

Dibromophakellstatin<smiles>O=C1c2ccccc2C(=O)N1CC=CBr</smiles>

(E,Z)-2-(3-bromoallyl)isoindoline-1,3-dione (11). ${ }^{\mathbf{S 8}}$ 1,3-Dibromo-1-propene (5.149 g, $25.76 \mathrm{mmol}, 1.0$ equiv) was added dropwise to a suspension of phthalimide potassium salt (4.77 g, $25.76 \mathrm{mmol}, 1.0$ equiv) and tetrabutylammonium bromide ( $415.2 \mathrm{mg}, 1.29 \mathrm{mmol}, 0.05$ equiv) in anhydrous $\mathrm{N}, \mathrm{N}$-dimethylformamide $(70 \mathrm{~mL})$ at $23{ }^{\circ} \mathrm{C} .{ }^{\mathrm{S} 9}$ After stirring for $16 \mathrm{~h}$, the solvent was removed and the residue was dissolved in methylene chloride $(300 \mathrm{~mL})$, washed with water $(200 \mathrm{~mL} \times 3)$, dried with anhydrous sodium sulfate, concentrated and recrystallized from ethyl acetate-hexanes to give $\mathbf{1 1}$ as white crystalline solid (6.256 g, 91\% trans:cis $=2: 3): R_{f}=0.29(20 \%$ ethyl acetate-hexane); FTIR (neat, $\mathrm{cm}^{-1}$ ) 1771, 1712, 1467, 1426, 1391, 1350, 1290, 1188, 1110, 946; $\left.{ }^{1} \mathrm{H} \mathrm{NMR} \mathrm{(400} \mathrm{MHz,} \mathrm{CDCl}_{3}\right)$ $\delta$ 7.88-7.86 (m, 2H), 7.74-7.72 (m, 2H), $6.47(\mathrm{dt}, J=13.7,1.0 \mathrm{~Hz}, 1 \mathrm{H}$ trans) , 6.39 (dt, $J=7.2,1.7 \mathrm{~Hz}$, $1 \mathrm{H}$ cis), 6.29 (dt, $J=13.6,6.8 \mathrm{~Hz}, 1 \mathrm{H}$ trans), 6.24 (dt, $J=7.2,6.2 \mathrm{~Hz}, 1 \mathrm{H} \mathrm{cis}), 4.47$ (dd, $J=6.1,1.7 \mathrm{~Hz}$, $2 \mathrm{H}$ cis $), 4.25(\mathrm{dd}, J=6.8,1.1 \mathrm{~Hz}, 2 \mathrm{H}$ trans $) ;{ }^{13} \mathrm{C} \mathrm{NMR}\left(75 \mathrm{MHz}, \mathrm{CDCl}_{3}\right) \delta 167.9,167.7,134.3(2)$, 132.2, 132.1, 130.9, 129.1, 123.6 (2), 111.0 (2), 39.1, 37.6; $\mathrm{MS}\left(\mathrm{ES}^{+}\right)$calcd for $\mathrm{C}_{11} \mathrm{H}_{8} \mathrm{BrNO}_{2}(\mathrm{M}+\mathrm{H})^{+}$ 265.97, found 265.80. 


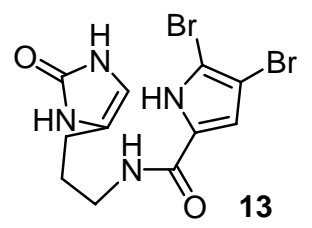

4,5-Dibromo- $N$-(3-(2,3-dihydro-2-oxo- $1 H$-imidazol-4-yl)propyl)-1H-pyrrole-2carboxamide (13). Imidazol-2-one (1) $(840.8 \mathrm{mg}, 10 \mathrm{mmol}, 10$ equiv), vinyl bromide 11 (266.1 mg, $1.0 \mathrm{mmol}, 1.0$ equiv) and sodium acetate trihydrate (480.2 $\mathrm{mg}, 3.0 \mathrm{mmol}, 3$ equiv) were vacuum-dried and filled with argon for 3 times before degassed dimethyl sulfoxide $(9 \mathrm{~mL})$ was introduced. After stirring at $80^{\circ} \mathrm{C}$ in dark for $10 \mathrm{~min}$, a solution of palladium acetate $(44.9 \mathrm{mg}, 0.2 \mathrm{mmol}, 0.2$ equiv) in degassed dimethyl sulfoxide $(1.0 \mathrm{~mL})$ was quickly added. The reaction was stirred at $80^{\circ} \mathrm{C}$ in dark for $2 \mathrm{~h}$ and quenched by pouring into water $(100 \mathrm{~mL})$. The mixture was filtered through a short pad of RP-C18 silica gel and washed with $10 \%$ methanol- $\mathrm{H}_{2} \mathrm{O}(100 \mathrm{~mL})$ followed by $N, N$-dimethylformamide $(100-120 \mathrm{~mL})$. The $N, N$-dimethylformamide wash portion was collected and used directly for the next step.

The $N, N$-dimethylformamide portion from the above step was dilute with methanol $(120 \mathrm{~mL})$ and was hydrogenated under hydrogen $(1 \mathrm{~atm})$ at room temperature for $12 \mathrm{~h}$. The solution was filtered and the filtrate was concentrated to dryness. The solid was washed with methylene chloride $(3 \mathrm{~mL} \times 3)$, and concentrated to give 2-(3-(2,3-dihydro-2-oxo- $1 H$-imidazol-5-yl)propyl)isoindoline-1,3-dione: $R_{f}=0.43$ (10\% methanol-methylene chloride); FTIR (neat, $\left.\mathrm{cm}^{-1}\right) 3401,3307,1996,1766,1710,1631,1552$, $1402, ;{ }^{1} \mathrm{H}$ NMR $\left(400 \mathrm{MHz}, \mathrm{DMSO}-d_{6}\right) \delta 9.70(\mathrm{~s}, 1 \mathrm{H}), 9.38(\mathrm{~s}, 1 \mathrm{H}), 7.88-7.82(\mathrm{~m}, 4 \mathrm{H}), 5.95(\mathrm{~s}, 1 \mathrm{H})$, $3.57(\mathrm{t}, J=6.8 \mathrm{~Hz}), 2.24(\mathrm{t}, J=7.5 \mathrm{~Hz}), 1.82-1.74(\mathrm{~m}, 2 \mathrm{H}) ;{ }^{13} \mathrm{C}$ NMR $\left(75 \mathrm{MHz}\right.$, DMSO- $\left.d_{6}\right) \delta 168.0$, 154.9, 134.4, 131.6, 123.0, 121.0, 103.9, 37.0, 26.5, 22.7; $\mathrm{MS}\left(\mathrm{ES}^{+}\right)$calcd for $\mathrm{C}_{14} \mathrm{H}_{13} \mathrm{~N}_{3} \mathrm{O}_{3}(\mathrm{M}+\mathrm{H})^{+}$ 272.10 , found 272.00 .

The 2-(3-(2,3-dihydro-2-oxo- $1 H$-imidazol-5-yl)propyl)isoindoline-1,3-dione obtained above was suspended in methanol $(200 \mathrm{~mL})$ and heated at $75^{\circ} \mathrm{C}$ for $30 \mathrm{~min}$. The remaining palladium was filtered off to afford a clear orange solution. Anhydrous hydrazine $(1.28 \mathrm{~mL}, 40 \mathrm{mmol}, \rightarrow 0.2 \mathrm{M}$ in methanol) was then added dropwise to this solution at $75^{\circ} \mathrm{C}$. After heating at $75^{\circ} \mathrm{C}$ for $1 \mathrm{~h}$, the yellow solution was concentrated, azeotroped with ethanol $(20 \mathrm{~mL} \times 2)$ and dried under vacuum for $2 \mathrm{~h}$. The resulting solid was then dissolved in deionized water $(25 \mathrm{~mL})$, and stirred with activated Dianion WA30 resin $(10 \mathrm{~mL})$ until the aqueous phase became clear and colorless. The mixture was filtered and the resin was washed with deionized water $(5 \mathrm{~mL} \times 2)$. The aqueous solution was concentrated, azeotroped with toluene $(5 \mathrm{~mL} \times 3)$ and dried under vacuum for $6 \mathrm{~h}$ to give 5 -(3-aminopropyl)- $1 \mathrm{H}$ imidazol-2(3H)-one as a yellow oil, which was used directly for the next step.

The 5-(3-aminopropyl)- $1 H$-imidazol-2(3H)-one obtained above ( $64.0 \mathrm{mg}, 0.453 \mathrm{mmol}, 1.0$ equiv), 4,5dibromo-2- (trichloroacetyl)pyrrole ${ }^{\mathrm{S} 12}(247.8 \mathrm{mg}, 0.669 \mathrm{mmol}, 1.5$ equiv) was dissolved in anhydrous $N, N$-dimethylformamide $(8 \mathrm{~mL})$. Triethylamine $(0.19 \mathrm{~mL}, 1.34 \mathrm{mmol}, 3.0$ equiv) was added and the solution was stirred at $60^{\circ} \mathrm{C}$ under argon in dark for $3 \mathrm{~h}$. The solvent was then removed and the residue was triturated with ether $(3 \mathrm{ml} \times 4)$. The solid was washed with $\mathrm{HCl}(0.1 \mathrm{~N}, 10 \mathrm{~mL})$, water and dried under vacuum in the presence of phosphorus pentoxideto give 13 as off-white solid (159.8 $\mathrm{mg}$, $41 \%$ over 4 steps): $R_{f}=0.15$ ( $10 \%$ methanol-methylene chloride); FTIR (neat, $\left.\mathrm{cm}^{-1}\right) 3401,3307,1996$, 1681, 1643, 1531, 1416; ${ }^{1} \mathrm{H}$ NMR (400 MHz, DMSO- $\left.d_{6}\right) \delta 12.67$ (s, 1H), $9.75(\mathrm{~s}, 1 \mathrm{H}), 9.42(\mathrm{~s}, 1 \mathrm{H})$, $8.12(\mathrm{t}, J=5.6 \mathrm{~Hz}, 1 \mathrm{H}), 6.91(\mathrm{~d}, J=2.7 \mathrm{~Hz}, 1 \mathrm{H}), 5.97(\mathrm{~s}, 1 \mathrm{H}), 3.20(\mathrm{dt}, J=6.8,6.4 \mathrm{~Hz}, 2 \mathrm{H}), 2.24(\mathrm{t}, J$ $=7.3 \mathrm{~Hz}, 2 \mathrm{H}), 1.67(\mathrm{tt}, J=7.3,6.8 \mathrm{~Hz}, 2 \mathrm{H}) ;{ }^{13} \mathrm{C}$ NMR $\left(100 \mathrm{MHz}\right.$, DMSO- $\left.d_{6}\right) \delta 158.9,154.9,128.2$, 121.4, 112.4, 104.4, 103.7, 97.8, 38.0, 27.7, 22.6; $\mathrm{MS}\left(\mathrm{ES}^{+}\right)$calcd for $\mathrm{C}_{11} \mathrm{H}_{12} \mathrm{Br}_{2} \mathrm{~N}_{4} \mathrm{O}_{2}(\mathrm{M}+\mathrm{H})^{+}$390.93, found 391.20 .

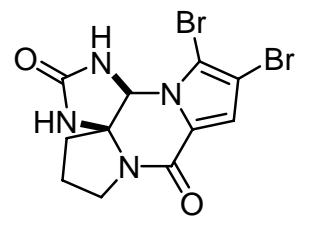

( \pm )-Dibromophakellstatin. Imidazolinone 13 (50 $\mathrm{mg}, 0.128 \mathrm{mmol}, 1.0$ equiv) was suspended in 2,2,2-trifluoroethanol $(5 \mathrm{~mL})$ (sonicate the mixture to give fine suspension if necessary) and anhydrous sodium carbonate (136.4 $\mathrm{mg}, 1.28 \mathrm{mmol}$, 10 equiv), iodobenzene diacetate ( $41.1 \mathrm{mg}, 0.128 \mathrm{mmol}, 1.0$ equiv) was added at 23 ${ }^{\circ} \mathrm{C}$. The reaction mixture became clear after several min. After stirring for $1 \mathrm{~h}$, sodium carbonate was filtered off and the filtrate was concentrated to nearly dryness. With stirring, $\mathrm{HCl}(0.1 \mathrm{~N}, 5 \mathrm{~mL})$ was added dropwise to the resulting residue. After stirring for $10 \mathrm{~min}$, the 
precipitate was collected by filtration, washed with water, and dried under vacuum in the presence of phosphorus pentoxide to give dibromophakellstatin as off-white solid (48.3 mg, 97\%): $R_{f}=0.50(10 \%$ methanol-methylene chloride); FTIR (neat, $\mathrm{cm}^{-1}$ ) 3401, 3306, 1996, 1722, 1641, 1452; ${ }^{1} \mathrm{H}$ NMR (400 MHz, DMSO- $\left.d_{6}\right) \delta 8.27(\mathrm{~s}, 1 \mathrm{H}), 7.98(\mathrm{~s}, 1 \mathrm{H}), 6.91(\mathrm{~s}, 1 \mathrm{H}), 5.99(\mathrm{~s}, 1 \mathrm{H}), 3.58-3.53(\mathrm{~m}, 1 \mathrm{H}), 3.46-3.39$ $(\mathrm{m}, 1 \mathrm{H}), 2.32-2.26(\mathrm{~m}, 1 \mathrm{H}), 2.18-2.04(\mathrm{~m}, 2 \mathrm{H}), 2.00-1.94(\mathrm{~m}, 1 \mathrm{H}) ;{ }^{13} \mathrm{C}$ NMR $\left(100 \mathrm{MHz}\right.$, DMSO- $\left.d_{6}\right) \delta$ $\left.157.9,154.1,125.4,113.8,105.6,101.2,79.0,68.6,44.2,40.4,18.9 ; \mathrm{MS} \mathrm{ES}^{+}\right)$calcd for $\mathrm{C}_{11} \mathrm{H}_{10} \mathrm{Br}_{2} \mathrm{~N}_{4} \mathrm{O}_{2}(\mathrm{M}+\mathrm{H})^{+} 388.92$, found 388.70 .

S1 Jaguar, version 6.5, Schrödinger, LLC, New York, NY, 2006.

Hay, P. J.; Wadt, W. R. J. Chem. Phys. 1985, 82, 299.

Keith, J. A.; Oxgaard, J.; Goddard, III, W. A. J. Am. Chem. Soc. 2006, 128, 3132-3133.

Tannor, D. J.; Marten, B.; Murphy, R.; Friesner, R. A.; Sitkoff, D.; Nicholls, A.; Ringnalda, M.;

Goddard, W. A., III; Honig, B. J. Am. Chem. Soc. 1994, 116, 11875-11872.

Marten, B.; Kim, K.; Cortis, C.; Friesner, R. A.; Murphy, R. B.; Ringnalda, M. N.; Sitkoff, D.; Honig, B. J. Phys. Chem. 1996, 100, 11775-11788.

Morrill, C.; Grubbs, R. H. J. Org. Chem. 2003, 68, 6031-6034.

${ }^{510}$ Xu, W.; Abboud, K. A.; Ghiviriga, I.; Dolbier, Jr., W. R.; Rapp, M.; Wnuk, S. Org. Lett. 2006, 8, $5549-5551$.

S11 Holden, K. G.; Mattson, M. N.; Cha,K. H.; Rapoport, H. J. Org. Chem. 2002, 67, 5913-5918.

S12 Linington, R. G.; Williams, D. E.; Tahir, A.; van Soest, R.; Andersen, R. J. Org. Lett. 2003, 5, $2735-2738$. 

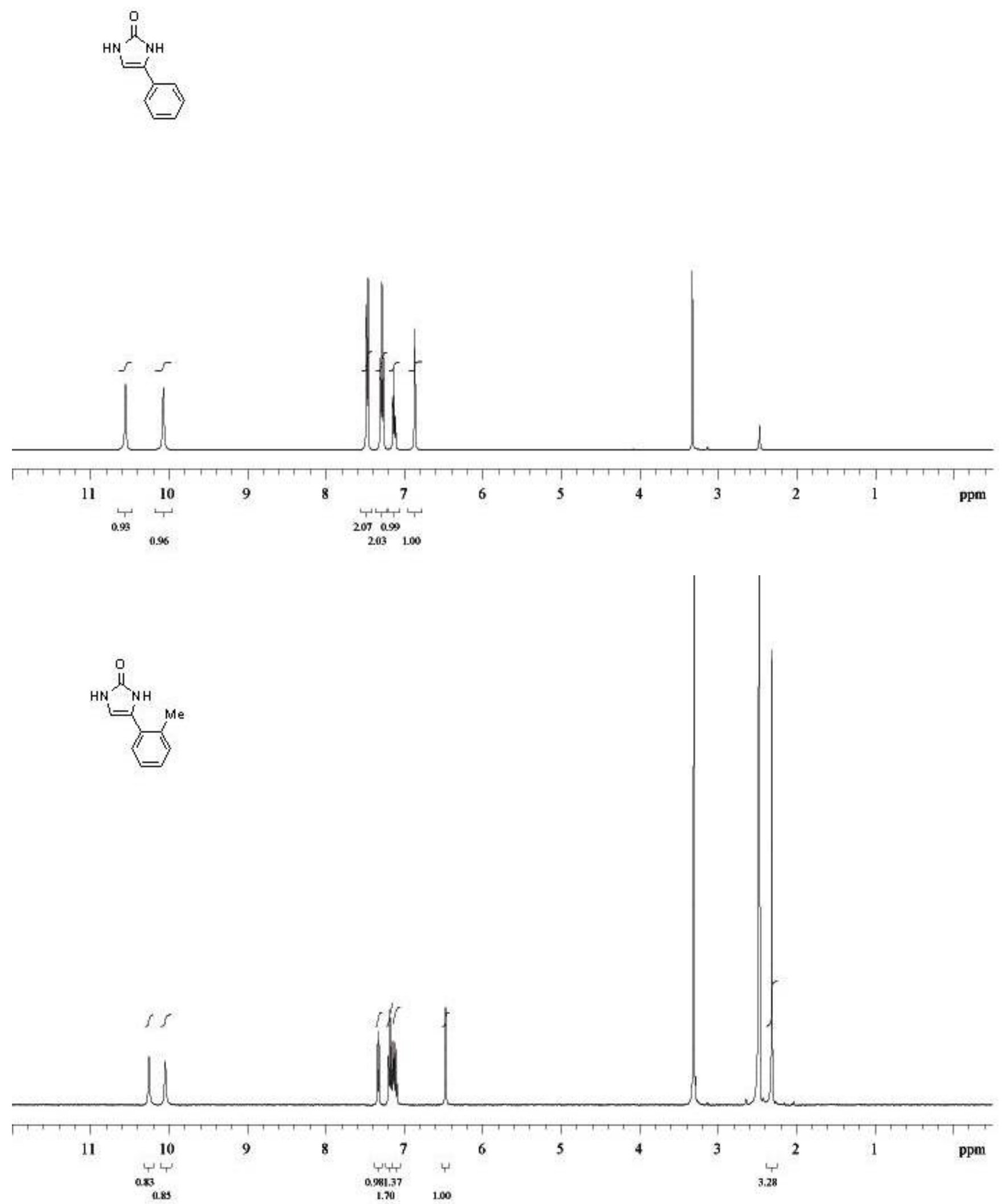


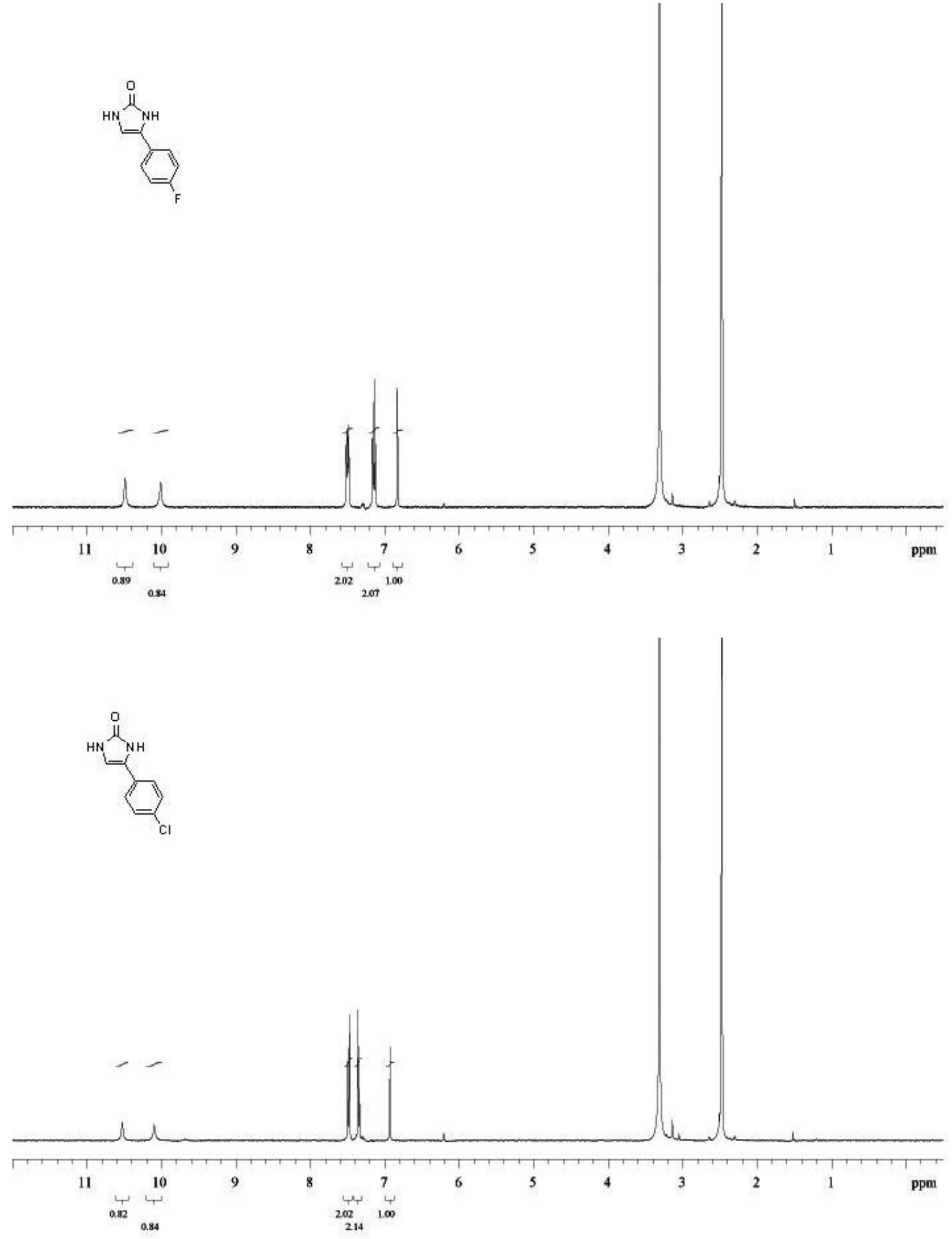




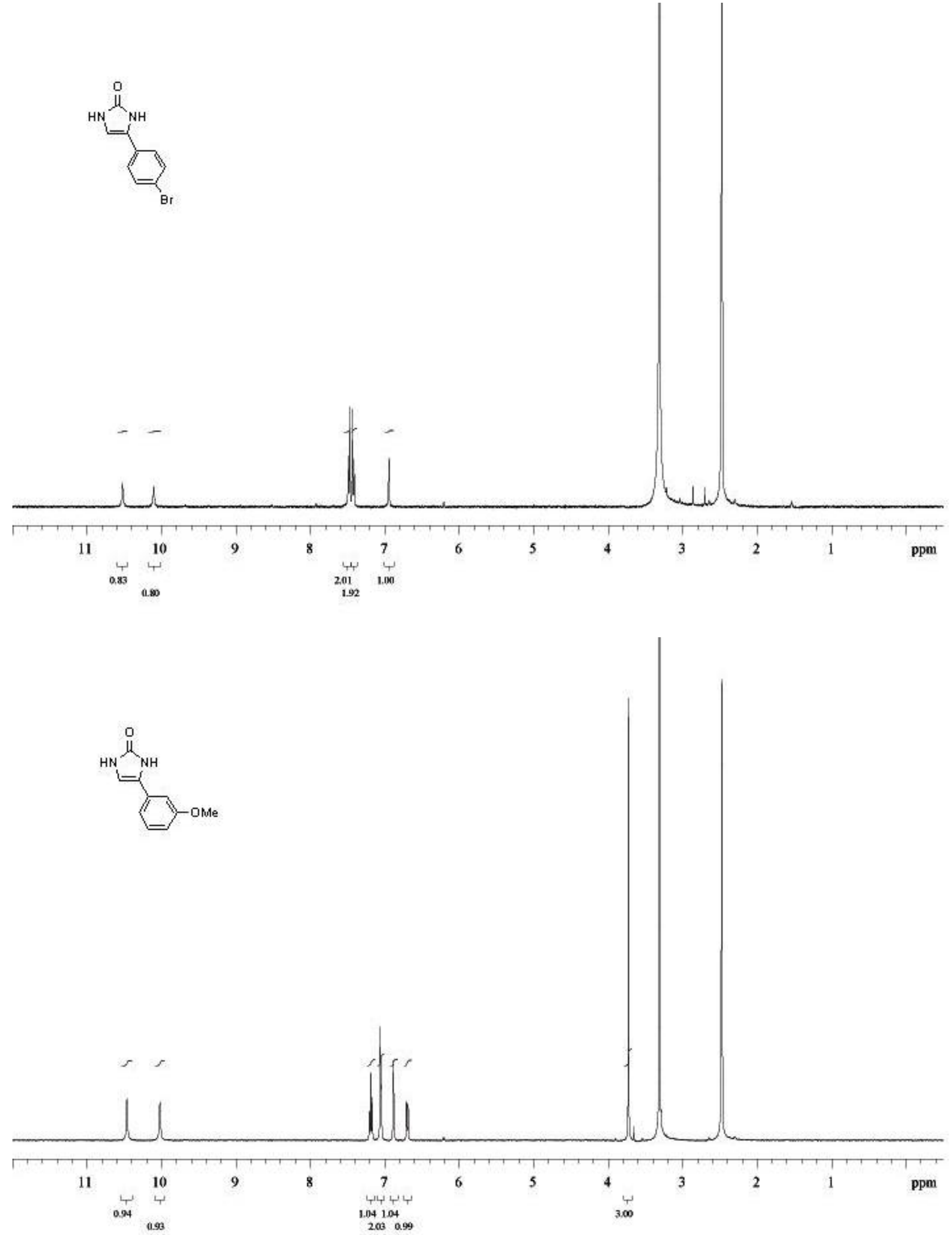



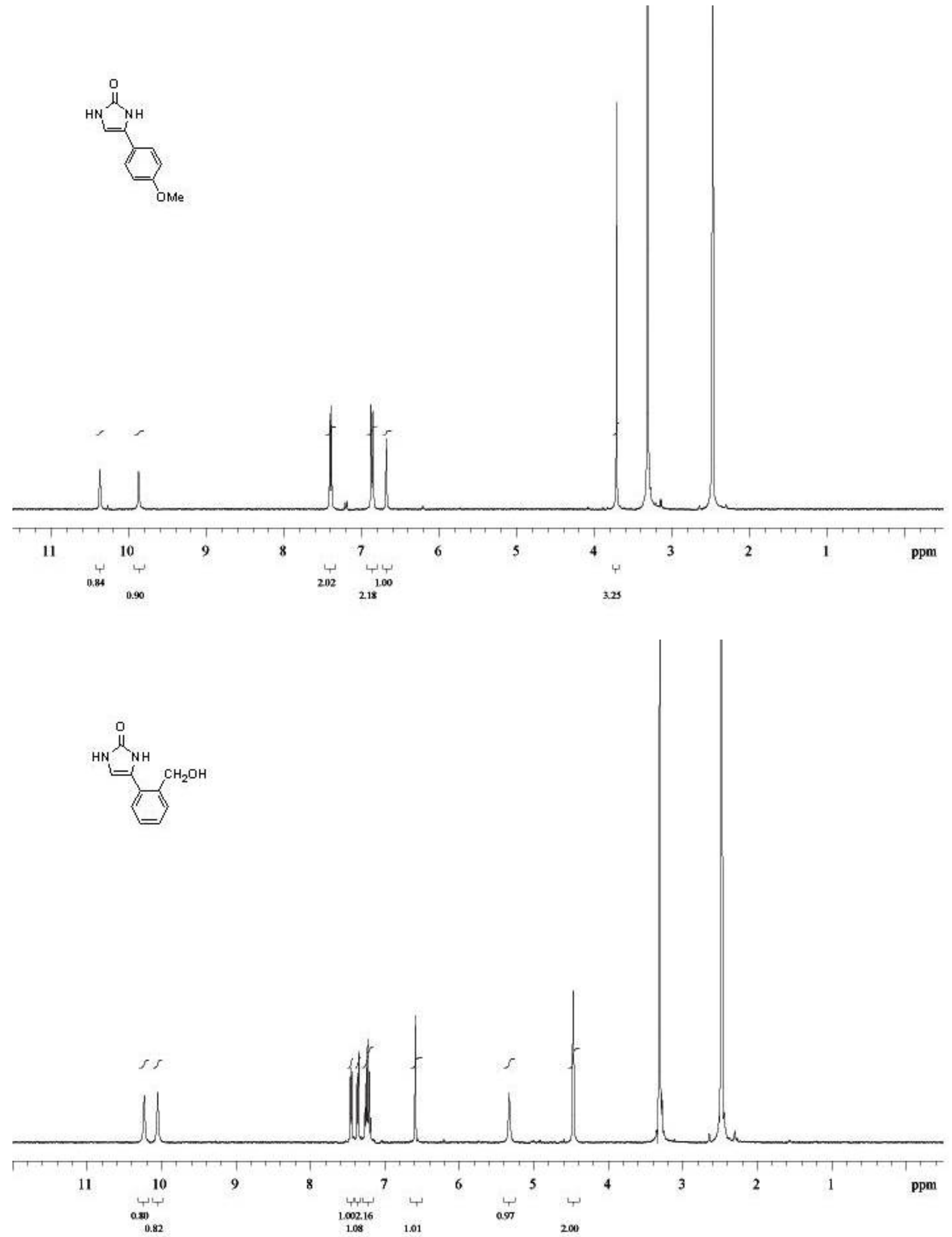

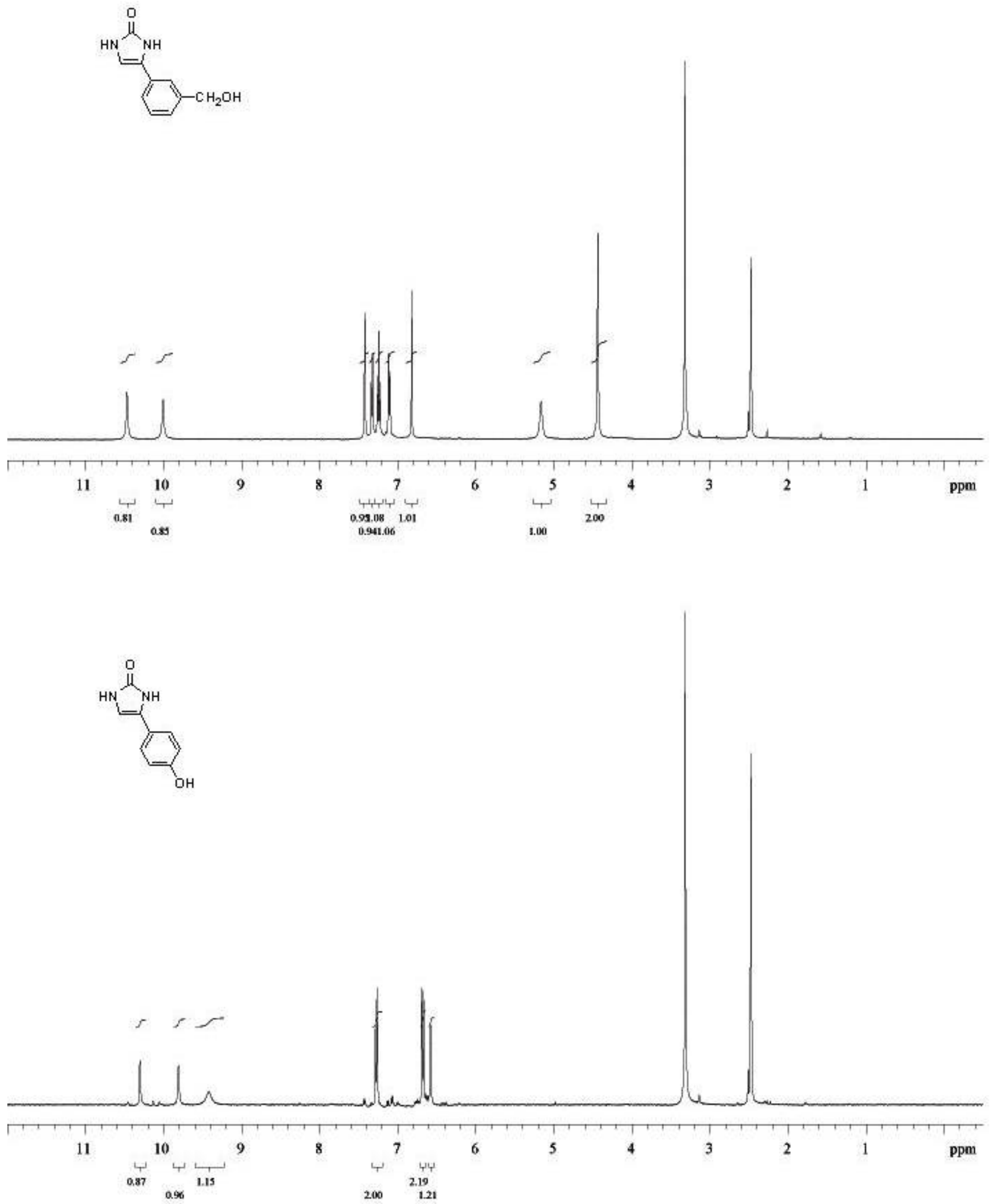


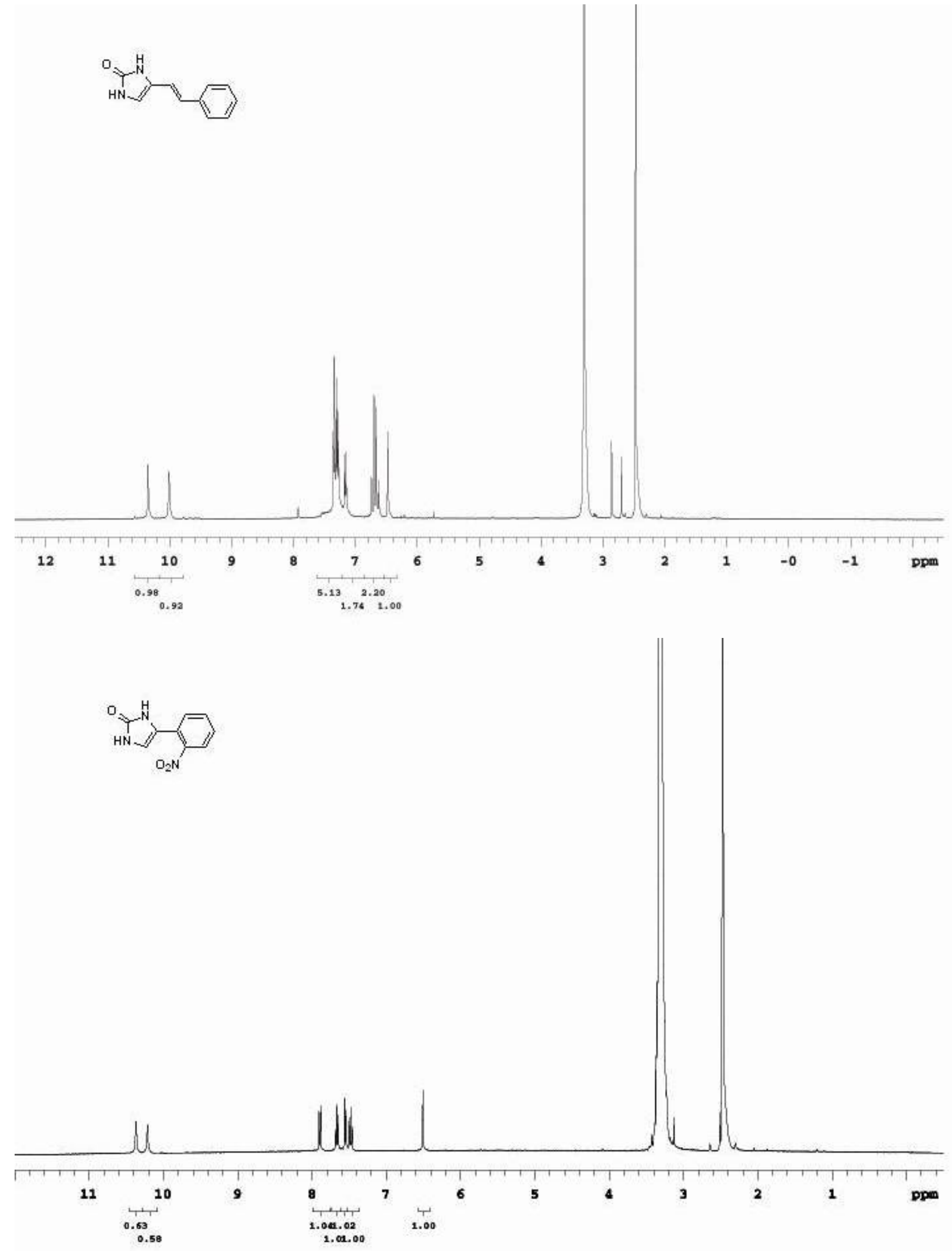




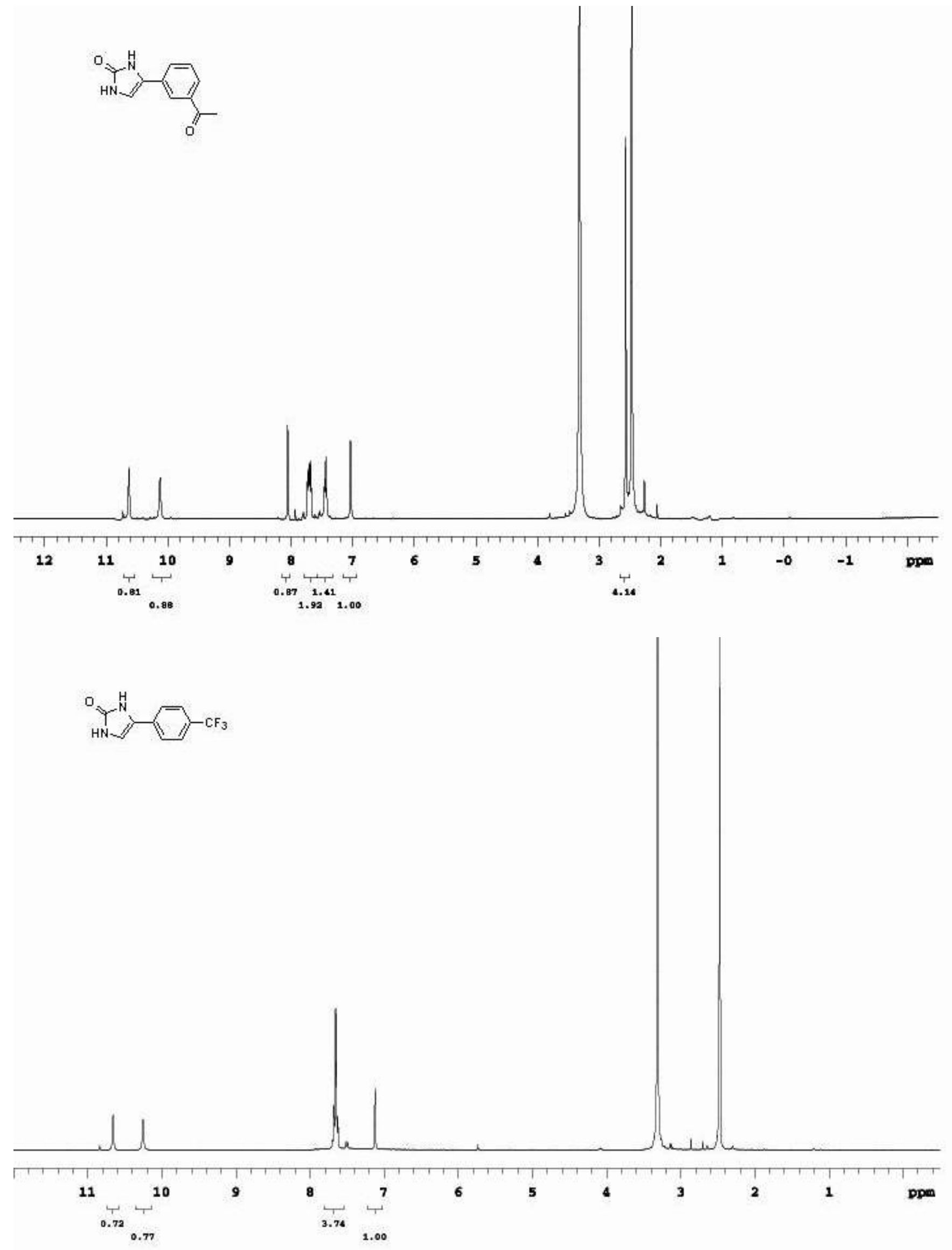




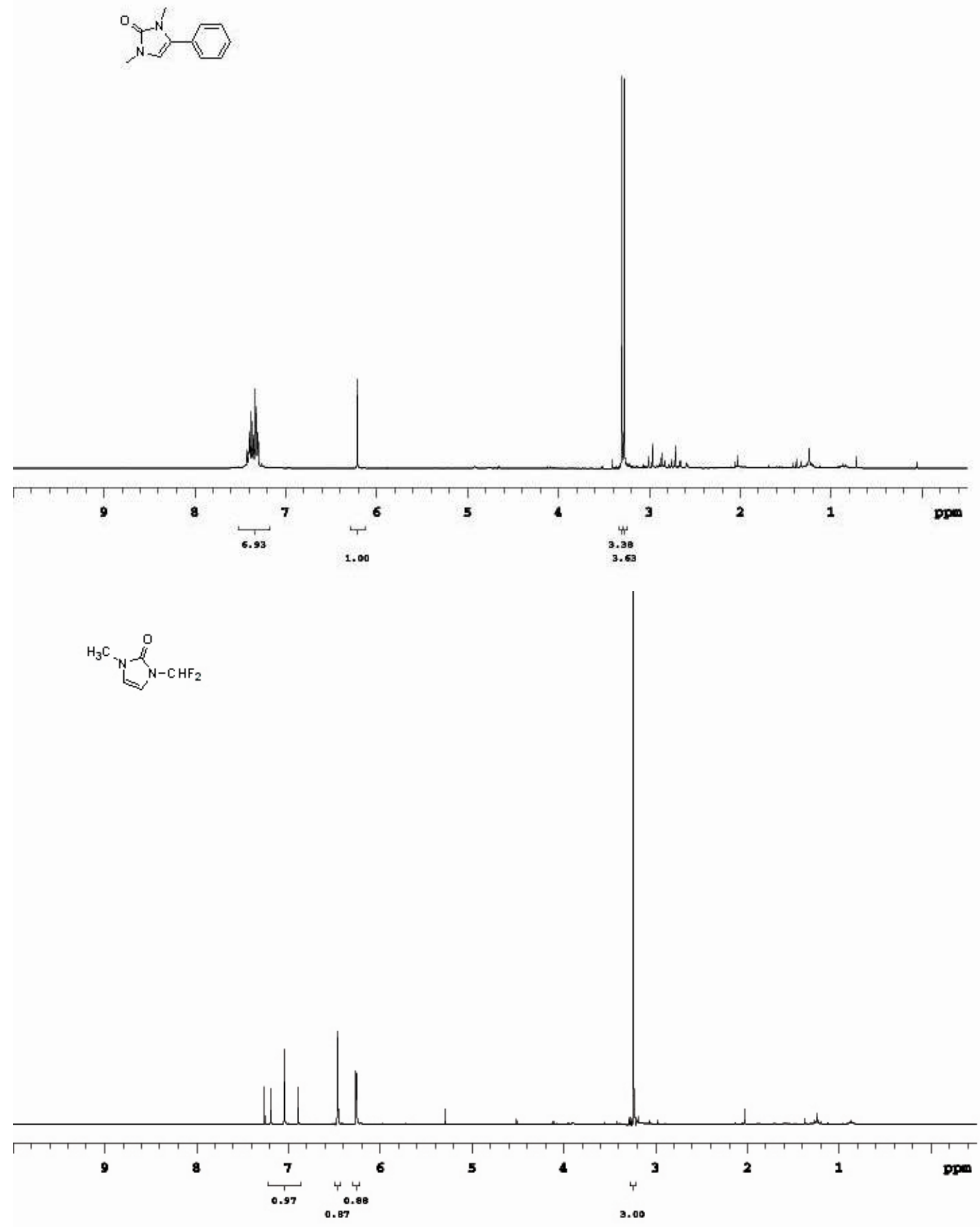



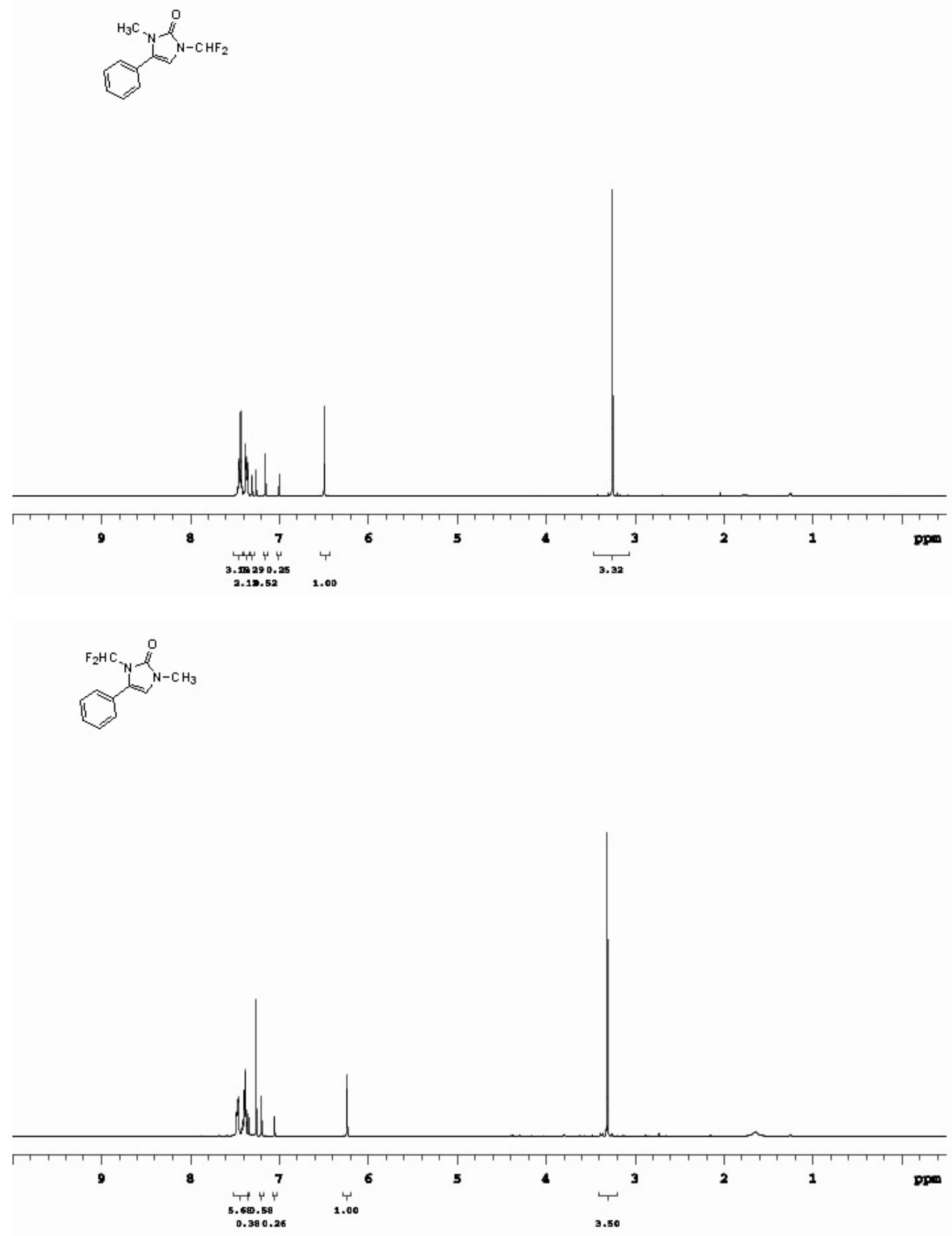


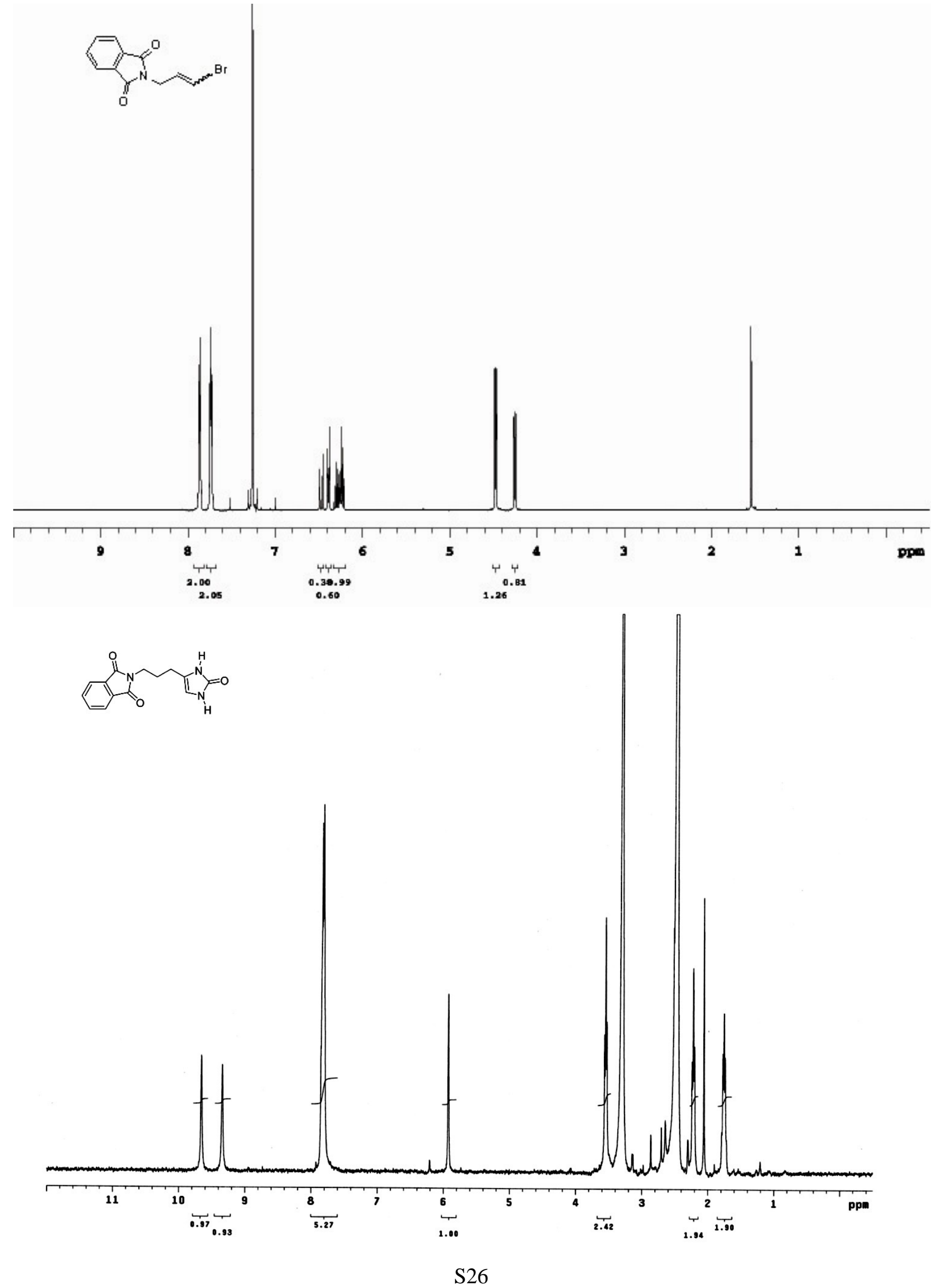


<smiles>O=C(NCCCc1c[nH]c(=O)[nH]1)c1cc(Br)c(Br)[nH]1</smiles>
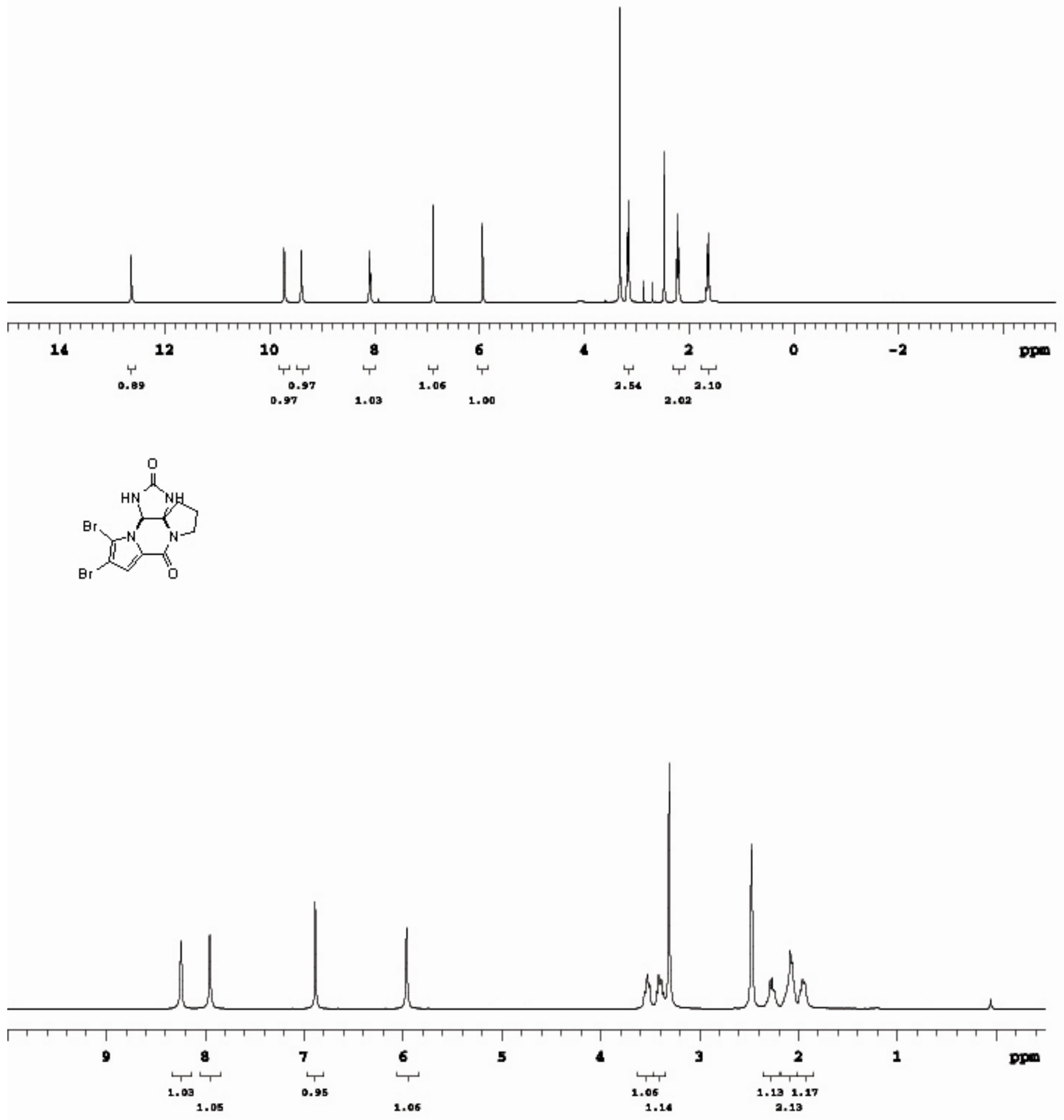

S27 